\title{
THE GEOMETRY OF THE SPACE OF HOLOMORPHIC MAPS FROM A RIEMANN SURFACE TO A COMPLEX PROJECTIVE SPACE
}

\author{
SADOK KALLEL \& R. JAMES MILGRAM
}

\section{Introduction}

In recent years there have been a number of papers on the homology and geometry of spaces of holomorphic maps of the Riemann sphere into complex varieties, [21], [5], [17], [18], [3], [9]. However, the very classic question of the structure of the spaces of holomorphic maps from complex curves $M_{g}$ of genus $g \geq 1$ to complex varieties has proved to be very difficult. There is Segal's stability theorem, [21], which shows that the natural inclusion of the space of based holomorphic maps of degree $k$ into the space of all based maps $\operatorname{Hol}_{k}^{*}\left(M_{g}, V\right) \hookrightarrow \operatorname{Map}_{k}^{*}\left(M_{g}, V\right)$ is a homotopy equivalence through a range of dimensions which increases with $k$ when $V=\mathbf{P}^{n}$, the complex projective space. Also, there is the extension of this result by $\mathrm{J}$. Hurtubise to further $V$; [11]. But that is about all.

In this paper we begin the detailed study of the topology of the $H_{o l}^{*}\left(M_{g}, \mathbf{P}^{n}\right)$. We are able to completely determine these spaces and their homology when $M_{g}$ is an elliptic curve, and we give an essentially complete determination in the case where $M_{g}$ is hyperelliptic. In particular we determine the rational homology of these spaces when $k \geq 2 g-1$ in the elliptic and hyperelliptic cases.

Let $M_{g}$ be a genus $g$ complex curve. The key analytic result on the structure of $\mathrm{Hol}_{k}^{*}\left(M_{g}, \mathbf{P}^{1}\right)$ is Abel's Theorem which identifies the

Received June 22, 1996. The first author holds a Postdoctoral fellowship with CRM, Montréal, and the second author was partially supported by a grant from the NSF and a grant from the CNRS. 
disjoint pairs of $k$-tuples of unordered points $\left\langle r_{1}, \ldots, r_{k}\right\rangle$ and $\left\langle p_{1}, \ldots, p_{k}\right\rangle$ on $M_{g}$ that are the roots and poles of a meromorphic function on $M_{g}$ in terms of the Abel-Jacobi embedding of $M_{g}$ into its Jacobi variety, $\mu: M_{g} \rightarrow J\left(M_{g}\right)$. This extends to give necessary and sufficient conditions for an $(n+1)$ tuple $\left\{V_{i} \mid V_{i}=\left\langle x_{i, 1}, \ldots, x_{i, k}\right\rangle, i=0, \ldots, n\right\}$ of points in $M_{g}$ with $\cap_{0}^{n}\left\{V_{i}\right\}=\emptyset$ to be the root data for a holomorphic map of $M_{g}$ into $\mathbf{P}^{n}, n \geq 2$, and thus defines an embedding

$$
\operatorname{Hol}_{k}^{*}\left(M_{g}, \mathbf{P}^{n}\right) \subset\left(S P^{k}\left(M_{g}\right)\right)^{n+1},
$$

where $S P^{k}(X)$ is the $k$-fold symmetric product of $X$.

We begin by studying a compactification of the space $\operatorname{Hol}_{k}^{*}\left(M_{g}, \mathbf{P}^{n}\right)$, which we denote $E_{k}^{n}\left(M_{g}\right)$ obtained by taking the closure of the embedding

$$
H o l_{k}^{*}\left(M_{g}, \mathbf{P}^{n}\right) \subset\left(S P^{k}\left(M_{g}\right)\right)^{n+1}
$$

above. We show in (2.2) that $E_{k}^{n}\left(M_{g}\right)$ is given as the total space of a fibering,

$$
\left(\mathbf{P}^{k-g}\right)^{n+1} \longrightarrow E_{k}^{n}\left(M_{g}\right) \longrightarrow J\left(M_{g}\right)
$$

for $k \geq 2 g-1$, with $H^{*}\left(E_{k}^{n}\left(M_{g}\right) ; \mathbf{A}\right)=H^{*}\left(\mathbf{P}^{k-g}, \mathbf{A}\right)^{n+1} \otimes H^{*}\left(J\left(M_{g}\right) ; \mathbf{A}\right)$ for $\mathbf{A}$ any commutative ring of coefficients. Moreover, for $k \leq 2 g-2$, $E_{k}^{n}\left(M_{g}\right)$ is stratified by strata which are fiberings

$$
\left(\mathbf{P}^{s}\right)^{n+1} \rightarrow S_{k}^{s} \rightarrow A_{k}^{s}
$$

where the $A_{k}^{s}$ are subspaces of $J\left(M_{g}\right)$ determined by the curve. Specifically, $W_{k}^{j} \subset J\left(M_{g}\right)$ is the subspace of points in the image of the $k^{\text {th }}$ Abel-Jacobi map

$$
\mu_{k}: S P^{k}\left(M_{g}\right) \rightarrow J\left(M_{g}\right)
$$

with $\left(\mu_{k}\right)^{-1}(x)=\mathbf{P}^{s}$ where $s \geq \max (k-g, 0)+j$, and $A_{k}^{j}=W_{k}^{j}-W_{k}^{j+1}$. Both spaces $W_{k}^{j}$ and $A_{k}^{j}$ are extensively studied in [1], [10] (cf. §5).

This compactification has the property that $\operatorname{Hol}_{k}^{*}\left(M_{g}, \mathbf{P}^{n}\right)$ is open in $E_{k}^{n}\left(M_{g}\right)$, and we write $V_{k}^{n}\left(M_{g}\right)$ for the (closed) complement $E_{k}^{n}\left(M_{g}\right)$ $H_{o l}^{*}\left(M_{g}, \mathbf{P}^{n}\right)$. The space $E_{k}^{n}\left(M_{g}\right)$ being a closed, compact manifold, Alexander-Poincaré duality now gives

$$
\tilde{H}^{2 k(n+1)-2 n g-*}\left(\operatorname{Hol}_{k}^{*}\left(M_{g}, \mathbf{P}^{n}\right) ; \mathbf{F}\right) \cong H_{*}\left(E_{k}^{n}\left(M_{g}\right), V_{k}^{n}\left(M_{g}\right) ; \mathbf{F}\right)
$$

for $k \geq 2 g-1$. This then indicates that one can understand $H o l_{k}^{*}\left(M_{g}, \mathbf{P}^{n}\right)$ by first studying $V_{k}^{n}\left(M_{g}\right)$. 
The space $V_{k}^{n}\left(M_{g}\right) \subset E_{k}^{n}\left(M_{g}\right)$ is the union of two pieces. The first, for $k>2 g-1$, is a subfibration $Z_{k}^{n}\left(M_{g}\right)$ of the form

$$
\left\{\bigcup_{i=0}^{n+1}\left(\mathbf{P}^{k-g}\right)^{i} \times \mathbf{P}^{k-g-1} \times\left(\mathbf{P}^{k-g}\right)^{n-i}\right\} \longrightarrow Z_{k}^{n}\left(M_{g}\right) \longrightarrow J\left(M_{g}\right)
$$

with analogous definitions of $Z_{k}^{n}\left(M_{g}\right)$ for $k \leq 2 g-1$ (see Definition 2.6). Moreover, it is easy to determine the relative cohomology groups, $H^{*}\left(E_{k}^{n}\left(M_{g}\right), Z_{k}^{n}\left(M_{g}\right) ; \mathbf{F}\right)$ for $k>2 g-1$, and not too difficult for $k \leq$ $2 g-2$, provided we know enough about the $W_{k}^{j}$.

In the case of hyperelliptic and elliptic curves the Riemann-Roch Theorem determines the $W_{k}^{j}$ explicitly. Moreover, a complete description of the $W_{k}^{j}$ for all curves of genus $\leq 6$ is given in [1, pp.206 - 211].

In $\S 5$ we study the way in which the $W_{k}^{i}$ determine

$$
H^{*}\left(E_{k}^{n}\left(M_{g}\right), Z_{k}^{n}\left(M_{g}\right) ; \mathbf{A}\right)
$$

in detail and obtain a spectral sequence converging to these groups with explicit $E^{1}$-term.

Proposition 5.9. Suppose that $k \leq g$. Then there is a spectral sequence converging to $H^{*}\left(E_{k}^{n}\left(M_{g}\right), Z_{k}^{n}\left(M_{g}\right) ; \mathbf{A}\right)$ with $E^{1}$-term

$$
\begin{aligned}
E^{1}= & H_{*}\left(S P^{k}\left(M_{g}\right), S P^{k-1}\left(M_{g}\right) ; \mathbf{A}\right) \\
& \oplus \coprod_{i} H_{*}\left(W_{k}^{i}, W_{k-1}^{i} ; \mathbf{A}\right) \otimes \tilde{H}^{*}\left(S^{2 i(n+1)} ; \mathbf{A}\right),
\end{aligned}
$$

and in case $k \geq g$ then

$$
\begin{aligned}
E^{1}= & \Sigma^{2(k-g)(n+1)} H_{*}\left(J\left(M_{g}\right), W_{k-1}^{k-g} ; \mathbf{A}\right) \\
& \oplus \coprod_{i>k-g} H_{*}\left(W_{k}^{i}, W_{k-1}^{i} ; \mathbf{A}\right) \otimes \tilde{H}_{*}\left(S^{2 i(n+1)} ; \mathbf{A}\right) .
\end{aligned}
$$

The second part of $V_{k}^{n}\left(M_{g}\right)$ consists of the image of an action map

$$
\nu: M_{g} \times E_{k-1}^{n}\left(M_{g}\right) \rightarrow E_{k}^{n}\left(M_{g}\right)
$$

introduced in (2.4) that puts in redundant roots, and is the main source of difficulty in recovering the homology of $\operatorname{Hol}_{k}^{*}\left(M_{g}, \mathbf{P}^{n}\right)$ from the homology groups $H_{*}\left(E_{k}^{n}\left(M_{g}\right) ; \mathbf{A}\right)$ or $H_{*}\left(E_{k}^{n}\left(M_{g}\right), Z_{k}^{n}\left(M_{g}\right) ; \mathbf{A}\right)$. In order to handle this part we construct a spectral sequence which converges to 
the homology of the pair $\left(E_{k}^{n}\left(M_{g}\right), V_{k}^{n}\left(M_{g}\right)\right)$ starting with the relative groups

$$
H_{*}\left(E_{k}^{n}\left(M_{g}\right), Z_{k}^{n}\left(M_{g}\right) ; \mathbf{F}\right)
$$

Theorem 4.3. There is a spectral sequence converging to

$$
H_{*}\left(E_{k}^{n}\left(M_{g}\right), V_{k}^{n}\left(M_{g}\right) ; \mathbf{F}\right)
$$

for any field $\mathbf{F}$ with $E^{1}$-term

$$
\begin{aligned}
E^{1}=\coprod_{\substack{i+j=k \\
i \geq 1}} H_{*} & \left(E_{i}^{n}\left(M_{g}\right), Z_{i}^{n}\left(M_{g}\right) ; \mathbf{F}\right) \\
& \otimes H_{*}\left(S P^{j}\left(\Sigma M_{g}\right), S P^{j-1}\left(\Sigma M_{g}\right) ; \mathbf{F}\right) \\
& \oplus H_{*}\left(\left(S P^{k}\left(\Sigma M_{g}\right), S P^{k-1}\left(\Sigma M_{g}\right) ; \mathbf{F}\right) .\right.
\end{aligned}
$$

The rest of $\S 4$ discusses the structure of $d_{1}$ and various properties such as the multiplicative pairing of the spectral sequences discussed immediately after the proof of (4.3). Of course the structure of the groups $H_{*}\left(S P^{j}\left(\Sigma M_{g}\right), S P^{j-1}\left(\Sigma M_{g}\right) ; \mathbf{F}\right)$ is well known from e.g. [7], [6], and [19]. It is also reviewed in $\S 6$.

Next we use these results to clarify the structure of the natural inclusions

$$
\operatorname{Hol}_{k}^{*}\left(M_{g}, \mathbf{P}^{n}\right) \hookrightarrow \operatorname{Map}_{k}^{*}\left(M_{g}, \mathbf{P}^{n}\right) .
$$

The spectral sequence of (4.3) has a natural break at $i=2 g-1$ in the sense that $i \geq 2 g-1$ implies that the relative homology groups

$$
H_{*}\left(E_{i}^{n}\left(M_{g}\right), Z_{i}^{n}\left(M_{g}\right) ; \mathbf{F}\right)=\left\{\begin{array}{l}
0 *<2(i-g)(n+1), \\
H_{*-2(i-g)(n+1)}\left(J\left(M_{g}\right) ; \mathbf{F}\right) \text { otherwise }
\end{array}\right.
$$

and, when $i<2 g-1$, the groups depend on the structure of $\mu_{i}$, the $W_{i}^{r}$, and have to be determined case by case. We call the case $i \geq 2 g-1$ the stable range for the spectral sequence and completely determine the differentials in this range. For $*>(2 n-1)(k-2 g+1)$ only the stable homology contributes to $H_{*}\left(E_{k}^{n}\left(M_{g}\right), V_{k}^{n}\left(M_{g}\right) ; \mathbf{F}\right)$. Thus, by Poincaré duality, the unstable range only contributes homology above this range. On the other hand it is easily seen that the duals of the stable range classes inject into $H_{*}\left(\operatorname{Map}_{k}^{*}\left(M_{g}, \mathbf{P}^{n}\right) ; \mathbf{F}\right)$, and many of these classes live considerably above the stable range above. Thus, as was the case with 
$H o l_{k}^{*}\left(\mathbf{P}^{1}, \mathbf{P}^{n}\right)([5])$ one has considerably more information about the map than was given by Segal's stability theorem.

In the final sections we study the cases of elliptic and hyperelliptic curves. Here, as indicated, the Riemann-Roch Theorem gives complete control of the $W_{k}^{r}$ and consequently the $E^{1}$-term of the spectral sequence is within range of calculation. In particular, for elliptic curves the situation is completely understood.

Typical of the results in the elliptic case is

Lemma 9.4. Let $I=\left(b_{1}, b_{2}\right)$ be the augmentation ideal in the polynomial ring $\mathbf{Q}\left[b_{1}, b_{2}\right]$ where $\operatorname{dim}\left(b_{i}\right)=2, i=1,2$. Then

$$
\begin{aligned}
H_{*}\left(H o l_{k}^{*}\left(M_{1}, \mathbf{P}^{1}\right) ; \mathbf{Q}\right) \simeq\{ & \left.\mathbf{Q}\left[b_{1}, b_{2}\right] / I^{k+1}\right\}\left(1, e_{1}, e_{2}, h_{1}, h_{2}, v\right) \\
& \oplus \mathbf{Q}\left(w_{1}, w_{2}, \ldots, w_{2 k-1}\right),
\end{aligned}
$$

where $\operatorname{dim}\left(e_{i}\right)=1, \operatorname{dim}\left(h_{i}\right)=2, \operatorname{dim}\left(w_{i}\right)=2 k-3$ and $\operatorname{dim}(v)=3$.

Here $M_{1}$ is an arbitrary elliptic curve. Additionally, it turns out that the map

$$
H^{*}\left(\operatorname{Map}_{k}^{*}\left(\dot{M}_{1}, \mathbf{P}^{1}\right) ; \mathbf{Q}\right) \longrightarrow H^{*}\left(\operatorname{Hol}_{k}^{*}\left(M_{1}, \mathbf{P}^{1}\right) ; \mathbf{Q}\right)
$$

is surjective for all $k \geq 1$ in this case.

For hyperelliptic curves there are some technical questions which seem difficult to handle for finite field coefficients, but with some effort a complete determination of the $E^{1}$-term in (4.3) with Q-coefficients is given in $\S 10-15$, together with sufficient differentials to completely determine $E^{\infty}$ and conclude that $H_{*}\left(H_{o l} l_{k}^{*}\left(M_{g}, \mathbf{P}^{n}\right) ; \mathbf{Q}\right)$ injects into $H_{*}\left(\operatorname{Map}_{k}^{*}\left(M_{g}, \mathbf{P}^{n}\right) ; \mathbf{Q}\right)$ for $k \geq 2 g-1$ in the hyperelliptic case as well.

As the arguments are pretty involved we summarize the salient points here.

To begin, the Riemann-Roch Theorem gives a complete determination of the $W_{i}^{r}$ as quotients of $S P^{i}\left(M_{g}\right)$ via an action discussed in the proof of Lemma 10.2,

$$
S P^{r}\left(\mathbf{P}^{1}\right) \times S P^{l}\left(M_{g}\right) \longrightarrow S P^{l+2 r}\left(M_{g}\right)
$$

induced from the Abel-Jacobi map $\mu_{2}: S P^{2}\left(M_{g}\right) \rightarrow J\left(M_{g}\right)$ which fails to be an embedding at only one point where it has inverse image a copy of $\mathbf{P}^{1}$, the $\mathbf{P}^{1}$ in the action map above.

These observations give the following result for hyperelliptic curves: 
Lemma 10.2. Suppose that $M_{g}$ is hyperelliptic and $\tau \in J\left(M_{g}\right)$ is the hyperelliptic point. Let $k \geq 1$ and $t \leq\left[\frac{k}{2}\right]$. Then we have the following:

(a) for $k \leq g$, the space $W_{k}^{t}$ is $\mu_{k-2 t}\left(S P^{k-2 t}\left(M_{g}\right)\right)+t \tau$,

(b) for $2 g-1>k>g, t>k-g$, we also have

$$
W_{k}^{t}=\mu_{k-2 t}\left(S P^{k-2 t}\left(M_{g}\right)\right)+t \tau \text {. }
$$

Of course, this gives the $W_{k}^{t}$ as quotients, so, in order to obtain information about the $W_{k}^{t}$ we introduce some spectral sequences which take care of the details of the quotienting process in $\S 11$ through $\S 13$. Using them we are able to determine the rational homology of the $W_{k}^{t}$ as follows.

\section{Lemma 13.7.}

(a) The inclusion $W_{j} \subset J\left(M_{g}\right)$ induces an injection in rational homology $H_{*}\left(W_{j} ; \mathbf{Q}\right) \hookrightarrow H_{*}\left(J\left(M_{g}\right) ; \mathbf{Q}\right)=\Gamma\left(e_{1}, \ldots, e_{2 g}\right)$ with image the subvector space spanned by the subspaces

$$
\left\{\Gamma_{s}\left(e_{1}, \ldots, e_{2 g}\right)\left[M_{g}\right]^{t} \mid s+t \leq j\right\}
$$

where $\left[M_{g}\right]=\sum_{1}^{g} e_{2 i-1} e_{2 i}$ is the image of the fundamental class of $M_{g}$ under the Abel-Jacobi map $\mu_{*}$.

(b) $H_{*}\left(W_{j-1} ; \mathbf{Q}\right)$ injects into $H_{*}\left(W_{j} ; \mathbf{Q}\right)$ under the inclusion so the relative groups are given as

$$
H_{*}\left(W_{j}, W_{j-1} ; \mathbf{Q}\right) \cong H_{*}\left(W_{j} ; \mathbf{Q}\right) / H_{*}\left(W_{j-1} ; \mathbf{Q}\right) .
$$

Next we turn to the homology of the spaces $\operatorname{Hol}_{k}^{*}\left(M_{g}, \mathbf{P}^{n}\right)$ in the hyperelliptic cases. This involves using the spectral sequences and calculations above. Of course, the $E^{1}$-term in the spectral sequence (4.3) is very complex, but one is able to recognize in it the direct sum of a family of chain complexes, each of which calculates a part of a certain Tor or Ext group of the exterior algebra $\Gamma\left(e_{1}, \ldots, e_{2 g}\right)$ modulo the two sided ideal generated by $\left[M_{g}\right]$. This explains $\S 14$ which is devoted to the calculation of the relevant Tor-groups.

Finally, in $\S 15$ we are able to put these results together to obtain our main calculational result, 
Theorem 15.1. The natural map

$$
H_{*}\left(\operatorname{Hol}_{k}^{*}\left(M_{g}, \mathbf{P}^{n}\right) ; \mathbf{Q}\right) \rightarrow H_{*}\left(\operatorname{Map}_{k}^{*}\left(M_{g}, \mathbf{P}^{n}\right) ; \mathbf{Q}\right)
$$

is injective for $k \geq 2 g$ and $n>2$ if $M_{g}$ is hyperelliptic.

\section{Acknowledgements}

We would like to thank the "Centre de Recherches Mathématiques" at the University of Montréal for their support and hospitality during the period when much of this work was done. The special case of (based) meromorphic functions on the torus; i.e., $g=1, n=1$ was worked out as part of the first author's Ph.D thesis written under the direction of the second author. We also thank Professor J. Hurtubise for numerous comments and his generous support.

\section{Preliminaries on the Abel-Jacobi map}

We review some classical definitions and theorems about the algebraic geometry of curves. We begin by defining the Abel-Jacobi map together with the Jacobi variety $J\left(M_{g}\right)$ associated to any positive genus Riemann surface. Good references are [1] and [10].

Any Riemann surface $M_{g}$ of genus $g \geq 1$ has $g$ independent holomorphic sections of the cotangent bundle $\tau^{*}\left(M_{g}\right)$, (holomorphic 1-forms), $w_{1}, w_{2}, \ldots, w_{g}$, the abelian differentials on $M_{g}$.

Fix a basepoint $p_{0} \in M_{g}$. Then we can associate to each point $p \in M_{g}$ and each path $\gamma$ between $p_{0}$ and $p$ the vector of integrals

$$
\mu_{\gamma}(p)=\left(\int_{p_{0}}^{p} w_{1}, \ldots, \int_{p_{0}}^{p} w_{g}\right) \in \mathbf{C}^{g} .
$$

Since any two paths $\gamma$ and $\gamma^{\prime}$ between $p_{0}$ and $p$ together determine a closed loop based at $p_{0}$, (which we will denote as $L$ ), we have that $\mu_{\gamma}(p)$ is well defined up to vectors of the form

$$
\left(\int_{L} w_{1}, \ldots, \int_{L} w_{g}\right)
$$

If we choose a set of loops $L_{1}, \ldots, L_{2 g}$ which, in homology, form a basis 
for $H_{1}\left(M_{g} ; \mathbf{Z}\right)$, they give rise to the following $g \times 2 g$ period matrix

$$
\Omega=\left(\begin{array}{ccc}
\int_{L_{1}} w_{1} & \cdots & \int_{L_{2 g}} w_{1} \\
\vdots & \ddots & \vdots \\
\int_{L_{1}} w_{g} & \cdots & \int_{L_{2 g}} w_{g}
\end{array}\right)
$$

Thus, since the $w_{i}$ are closed, the values $\mu_{\gamma}(p)$ depend only on $p$ and not $\gamma$ in the quotient torus

$$
J\left(M_{g}\right)=\mathbf{C}^{g} / \Omega \cong\left(S^{1}\right)^{2 g} .
$$

Consequently, they give rise to a well defined map $\mu: M_{g} \rightarrow J\left(M_{g}\right)$ which is called the Abel-Jacobi map for $M_{g}$.

At this point we need to introduce symmetric products.

Definition. The $n$-fold symmetric product $S P^{n}(X)$ of the space $X$ is defined to be the set of unordered $n$-tuples of points of $X$; i.e.,

$$
S P^{n}(X)=X^{n} / \mathcal{S}_{n},
$$

where $\mathcal{S}_{n}$ is the symmetric group on $n$ letters. A point in $S P^{n}(X)$ will be written in the form $\sum m_{i} x_{i}$ with $m_{i}>0$ and $\sum m_{i}=n$, or in the form

$$
\left\langle x_{1}, x_{2}, \ldots, x_{n}\right\rangle \text {. }
$$

Remarks. Let $X$ be any $C W$ complex with base point *. Then there are inclusions $S P^{n}(X) \hookrightarrow S P^{n+1}(X)$ which identify $\sum_{i} n_{i} P_{i}$ with $\sum_{i} n_{i} P_{i}+*$, and we get the increasing sequence of spaces

$$
*=S P^{0}(X) \subset S P^{1}(X) \subset \cdots \subset S P^{n-1}(X) \subset S P^{n}(X) \subset \cdots .
$$

The union of this sequence is the infinite symmetric product $S P^{\infty}(X)$, based at *. Moreover, if $X$ is path connected, then the homotopy type of $S P^{\infty}(X)$ is independent of the choice of *.

We can extend the Abel-Jacobi map to the symmetric products of $M_{g}$ by the rule $\left\langle m_{1}, \ldots, m_{k}\right\rangle \mapsto \mu\left(m_{1}\right)+\cdots+\mu\left(m_{k}\right)$, obtaining the family of maps

$$
\mu_{k}: S P^{k}\left(M_{g}\right) \longrightarrow J\left(M_{g}\right)
$$

The map $\mu$ is called the Abel-Jacobi map and it, together with the extensions $\mu_{k}$, is a critical piece of the structure data for $M_{g}$. 
It is a remarkable result due to Andreotti that for any $M_{g}$ the symmetric products $S P^{k}\left(M_{g}\right)$ are all complex manifolds, indeed, complex algebraic varieties of complex dimension $k$. To see this note that the symmetric product $S P^{k}(\mathbf{C})$ is diffeomorphic to $\mathbf{C}^{k}$ via the map that takes the unordered collection $\left\langle z_{1}, \ldots, z_{k}\right\rangle$ of points in $\mathbf{C}$ to the coefficients of the monic polynomial of degree $k$ with the $z_{i}, 1 \leq i \leq k$ as roots. Since locally $S P^{k}\left(M_{g}\right)$ is modeled on $S P^{k}(\mathrm{C})$, the result follows.

Remark 1.2. In the special case that $M_{g}=\mathbf{P}^{1}$ a slight extension of the above argument identifies $S P^{k}\left(\mathbf{P}^{1}\right)$ with $\mathbf{P}^{k}$ which is now regarded as the space of all homogeneous polynomials of degree $k$ in 2 variables.

Remark 1.3. In (1.14) we point out an extension of this result due to Mattuck which for $k \geq 2 g-1$ identifies $S P^{k}\left(M_{g}\right)$ with the total space of a fibration over $\left(S^{1}\right)^{2 g}$ with fiber $\mathbf{P}^{k-g}$.

Remark 1.4. Another way of thinking about $J\left(M_{g}\right)$ is as the Picard group of $M_{g}$, the space of isomorphism classes of holomorphic line bundles on $M_{g}$. From this point of view the addition in $J\left(M_{g}\right)$ corresponds to the tensor product of line bundles. Under this correspondence, the map $\mu$ takes $m \in M_{g}$ to the line bundle obtained from the trivial bundle over $M_{g}$ by gluing in a copy of the negative Hopf bundle over $m$ (see [10]).

We can associate to every holomorphic function $f \in \operatorname{Hol}\left(M_{g}, \mathbf{P}^{1}\right)$ a divisor $(f)$ defined by $(f)=\sum n_{i} Z_{i}-\sum m_{j} P_{j}$ where $\left\{Z_{i}\right\},\left\{P_{i}\right\}$ are respectively the zeros and poles of $f$ with multiplicities $n_{i}$ and $m_{i}$ respectively. By standard residue calculations, it is easy to see that $\sum n_{i}=\sum m_{j}$. The space of pairs of disjoint divisors on a Riemann surface $(\zeta, \eta)$, subject to the condition $\operatorname{deg}(\zeta)=\operatorname{deg}(\eta)$, constitutes then the first step in the description of the space of holomorphic maps from the surface to the Riemann sphere $\mathbf{P}^{1}$.

For maps of $\mathbf{P}^{1}$ to itself this description is enough for there do exist meromorphic maps having prescribed roots and poles (of equidegree); i.e., any divisor $D=\zeta-\eta, \operatorname{deg} D=0$ is the divisor of a meromorphic function on $\mathbf{P}^{1}$. For the general case $g \geq 1$ it turns out that a pair $(\zeta, \eta)$ as above need not necessarily give rise to a meromorphic function and one needs a further condition.

Theorem 1.5 (Abel). Given positive divisors $D$ and $D^{\prime}$ on $M_{g}$, $\operatorname{deg} D=\operatorname{deg} D^{\prime}$, then there exists an $f \in \operatorname{Hol}\left(M_{g}, \mathbf{P}^{1}\right)$ so that $(f)=$ $D-D^{\prime}$ if and only if $\mu(D)=\mu\left(D^{\prime}\right)$. 
The $f$ associated to the difference $D-D^{\prime}$ is unique provided we specify in advance the image of $p_{0}$ (based maps) and neither $D$ nor $D^{\prime}$ contains the basepoint, $p_{0}$. Additionally, given $D-D^{\prime}$, there are unique disjoint positive divisors $D_{1}, D_{1}^{\prime}$ so that $D-D^{\prime}=D_{1}-D_{1}^{\prime}$ and any $f$ with $(f)=D-D^{\prime}$ will have roots precisely the terms in $D_{1}$ and poles in $D_{1}^{\prime}$. We make the following definition.

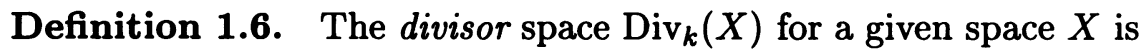
the set of pairs of disjoint positive divisors on $X$, i.e.,

$$
\operatorname{Div}_{k}(X)=\left\{\left(D, D^{\prime}\right) \in S P^{k}(X) \times S P^{k}(X) \mid D \cap D^{\prime}=\emptyset\right\}
$$

and more generally

$$
\begin{aligned}
\operatorname{Div}_{k}^{n}(X)=\{ & \left(D_{1}, \ldots, D_{n+1} \mid D_{i} \in S P^{k}(X), 1 \leq i \leq n+1\right. \\
& \left.D_{1} \cap D_{2} \cap \cdots \cap D_{n+1}=\emptyset\right\}
\end{aligned}
$$

Corollary 1.9. The space of based holomorphic maps of degree $k$, $\mathrm{Hol}_{k}^{*}\left(M_{g}, \mathbf{P}^{1}\right)$, is the inverse image of 0 under the subtraction map

$$
s: \operatorname{Div}_{k}\left(M_{g}-p_{0}\right) \longrightarrow J\left(M_{g}\right),
$$

where the subtraction map $s$ is given by $s\left(\left(D, D^{\prime}\right)\right)=\mu(D)-\mu\left(D^{\prime}\right)$.

More generally, and perhaps more usefully, we have

Corollary 1.10. The space of based holomorphic maps of degree $k$, $\mathrm{Hol}_{k}^{*}\left(M_{g}, \mathbf{P}^{n}\right)$ is the subspace of Div ${ }_{k}^{n}\left(M_{g}-p_{0}\right)$ consisting of $(n+1)$ tuples of degree $k$ positive divisors, subject to the following constraint:

$$
\mu_{k}\left(D_{1}\right)=\mu_{k}\left(D_{2}\right)=\cdots=\mu_{k}\left(D_{n+1}\right) .
$$

It was using this formulation of the space $\mathrm{Hol}_{k}^{*}\left(M_{g}, \mathbf{P}^{n}\right)$ that Segal [21] proceeded to prove his stability result. We will shortly give and use a yet more explicit version of this corollary. But before we do so, here are some of the standard results on the Abel-Jacobi map that we will be using:

(1.11) The orginal map $\mu: M_{g} \rightarrow J\left(M_{g}\right)$ is an embedding and the (complex) dimension of the image of $\mu_{d}: S P^{d}\left(M_{g}\right) \rightarrow J\left(M_{g}\right)$ is $d$ for $d \leq g$. In particular $\mu$ is onto for $d \geq g$ (Jacobi inversion theorem). 
(1.12) The preimage of any point $\mu(p) \in J\left(M_{g}\right), \mu_{d}^{-1}(\mu(p)) \in S P^{d}\left(M_{g}\right)$ is always a complex projective plane $\mathbf{P}^{m}$ for some $m \geq 0$.

(1.13) For $d \leq 2 g-2$ the dimension $m$ is less than or equal to $\frac{d}{2}$ (Clifford).

(1.14) For $d \geq 2 g-1$ the map $\mu_{d}$ makes $S P^{d}\left(M_{g}\right)$ into an analytic fiber bundle over $J\left(M_{g}\right)$ with fiber $\mathbf{P}^{d-g}$ (Mattuck [16]).

In $\S 9$ we will give more details on the structure of these maps for $g \leq 5$, but now we turn to the construction of an explicit model for the space $\operatorname{Hol}_{k}^{*}\left(M_{g}, \mathbf{P}^{n}\right)$ from our considerations thus far.

\section{A compactified version of $\operatorname{Hol}_{k}^{*}\left(M_{g}, \mathbf{P}^{n}\right)$}

It should be apparent from $\S 1$ that we are interested in the inverse image of 0 under $s: \operatorname{Div}_{k}\left(M_{g}-x_{0}\right) \rightarrow J\left(M_{g}\right)$ for holomorphic maps to $\mathbf{P}^{1}$, and generally in the inverse image of the iterated diagonal

$$
\Delta^{n+1}(J\left(M_{g}\right) \subset \underbrace{J\left(M_{g}\right) \times \cdots \times J\left(M_{g}\right)}_{n+1-\text { times }}
$$

under $\mu_{k} \times \cdots \times \mu_{k}$ for maps into $\mathbf{P}^{n}$.

Let the space $E_{i_{0}, i_{1}, \ldots, i_{n}}$ be defined as the fiber product of $\mu \times \cdots \times \mu$ and $\Delta$ in the diagram below

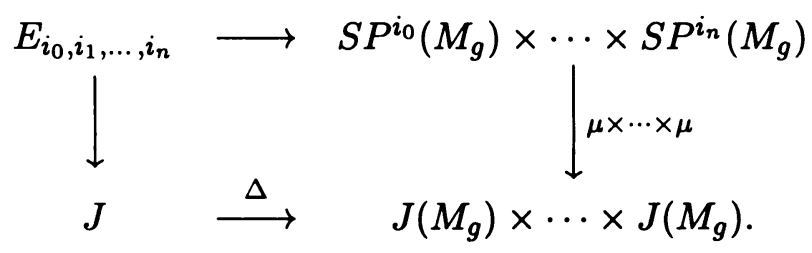

More explicitly

$$
\begin{aligned}
& E_{i_{0}, i_{1}, \ldots, i_{n}} \\
& \quad=\left\{\left(D_{0}, \ldots, D_{n}\right) \in S P^{i_{0}}\left(M_{g}\right) \times \cdots \times S P^{i_{n}}\left(M_{g}\right) \mid \mu\left(D_{i}\right)=\mu\left(D_{j}\right)\right\} .
\end{aligned}
$$

Clearly $\operatorname{Hol}_{k}^{*}\left(M_{g}, \mathbf{P}^{n}\right) \subset E_{k, k, \ldots, k}$ as the subset consisting of all the $\left\{\left(D_{0}, \ldots, D_{n}\right)\right\}$ where no $D_{i}$ contains $*$ and $\bigcap_{0}^{n} D_{i}=\emptyset$. Of course, for $k$ sufficiently small $\operatorname{Hol}_{k}^{*}\left(M_{g}, \mathbf{P}^{n}\right)$ is empty and this is consistent with the fact that Riemann surfaces of positive genus do not admit holomorphic functions with a single pole. However, once $\operatorname{Hol}_{k}^{*}\left(M_{g}, \mathbf{P}^{n}\right)$ is non-empty, and $k$ is sufficiently large, $\operatorname{Hol}_{k}^{*}\left(M_{g}, \mathbf{P}^{n}\right)$ will be open in 
$E_{k, \ldots, k}$ with $E_{k, \ldots, k}$ as its closure. Then $E_{k, k, \ldots, k}$ is the compactification of $\operatorname{Hol}_{k}^{*}\left(M_{g}, \mathbf{P}^{n}\right)$ mentioned in the title of this section.

Lemma 2.2. For $i_{j} \geq 2 g-1,0 \leq j \leq n$, we have a fibering

$$
\prod_{j=0}^{n} \mathbf{P}^{i_{j}-g} \longrightarrow E_{i_{0}, i_{1}, \ldots, i_{n}} \longrightarrow J\left(M_{g}\right)
$$

and the fiber is totally non-homologous to zero so

$$
H_{*}\left(E_{i_{0}, i_{1}, \ldots, i_{n}}\right) \cong H_{*}\left(\mathbf{P}^{i_{0}-g}\right) \otimes \cdots \otimes H_{*}\left(\mathbf{P}^{i_{n}-g}\right) \otimes H_{*}\left(J\left(M_{g}\right)\right) .
$$

Proof. In this range of dimensions $i_{j} \geq 2 g-1$, one can see by virtue of Mattuck's Theorem that the space $E_{i_{0}, i_{1}, \ldots, i_{n}}$ becomes the total space of a fibration

$$
\mathbf{P}^{i_{0}-g} \times \cdots \times \mathbf{P}^{i_{n}-g} \longrightarrow E_{i_{0}, i_{1}, \ldots, i_{n}} \longrightarrow J\left(M_{g}\right)
$$

obtained from the pull-back of a product of Mattuck's fibrations

$$
\mathbf{P}^{i_{0}-g} \times \cdots \times \mathbf{P}^{i_{n}-g} \longrightarrow S P^{i_{0}}\left(M_{g}\right) \times \ldots \times S P^{i_{n}}\left(M_{g}\right) \longrightarrow J\left(M_{g}\right)^{n+1}
$$

by the diagonal inclusion $\Delta$. Since $\Delta$ is injective and since the Serre spectral sequence for Mattuck's fibration collapses at $E_{2}$, the lemma follows from standard spectral sequence comparison arguments. q.e.d.

Further Properties of the $E_{i_{0}, \ldots, i_{n}}$

First of all, we notice that for any $j \geq 0$, we have natural inclusions

$$
E_{i_{0}, i_{1}, \ldots, i_{n}} \hookrightarrow E_{i_{0}, \ldots, i_{j}+1, \ldots, i_{n}}
$$

given by adding the basepoint in the $(j+1)^{s t}$ position.

Secondly we observe that the map

$$
\mu \times \cdots \times \mu: S P^{i_{0}}\left(M_{g}\right) \times \cdots \times S P^{i_{n}}\left(M_{g}\right) \rightarrow J\left(M_{g}\right)^{n+1}
$$

is multiplicative by construction, and so it induces a multiplicative pairing on the $E_{i_{0}, \ldots, i_{n}}$ which is commutative and associative:

$$
\nu^{I, J}: E_{i_{0}, \ldots, i_{n}} \times E_{j_{0}, \ldots, j_{n}} \longrightarrow E_{i_{0}+j_{0}, \ldots, i_{n}+j_{n}} .
$$

Also, $E_{1,1, \ldots, 1}=M_{g}$ and the pairing above thus yields an action

$$
\nu:\left(\coprod_{k=1}^{\infty} S P^{k}\left(M_{g}\right)\right) \times E_{i_{0}, \ldots, i_{n}} \longrightarrow \coprod_{k=1}^{\infty} E_{i_{0}+k, \ldots, i_{n}+k}
$$


explicitly defined via the diagonal multiplication

$$
\begin{gathered}
S P^{k}\left(M_{g}\right) \times\left(S P^{i_{0}}\left(M_{g}\right) \times \cdots \times S P^{i_{n}}\left(M_{g}\right)\right) \\
\longrightarrow S P^{i_{0}+k}\left(M_{g}\right) \times \cdots \times S P^{i_{n}+k}\left(M_{g}\right),
\end{gathered}
$$

which acts on points as follows

$$
\begin{aligned}
& \left(\sum m_{s},\left(\left\langle m_{11}, \ldots, m_{1, i_{0}}\right\rangle, \ldots,\left\langle m_{n 1}, \ldots, m_{n i_{n}}\right\rangle\right)\right) \\
& \quad \mapsto\left(\left\langle\sum m_{s}, m_{11}, \ldots, m_{1, i_{0}}\right\rangle, \ldots,\left\langle\sum m_{s}, m_{n 1}, \ldots, m_{n i_{n}}\right\rangle\right) .
\end{aligned}
$$

We can then give an explicit reformulation of the description of $\operatorname{Hol}_{i}^{*}\left(M_{g}, \mathbf{P}^{n}\right)$ in these terms.

Lemma 2.5. Let $\tilde{E}_{i_{0}, \ldots, i_{n}} \subset E_{i_{0}, \ldots, i_{n}}$ be the subspace in which no $m_{r s}$ is equal to the base point *. Then,

$$
\operatorname{Hol}_{i}^{*}\left(M_{g}, \mathbf{P}^{n}\right) \cong \tilde{E}_{i, i, \ldots, i}-\operatorname{Image}(\nu) \cap \tilde{E}_{i, i, \ldots, i}
$$

The following constructions are now needed for the remainder of our discussion.

Definition 2.6. For each $n$, the space $L E_{i}$ is the quotient

$$
L E_{i}=E_{i, i, \ldots, i} /\left\{\bigcup E_{i, i, \ldots, i-1, i, \ldots, i}\right\} \text {. }
$$

The space $Q E_{i}$ is the quotient

$$
L E_{i} /\{\operatorname{Image}(\nu)\}
$$

Remark 2.7. When $i \geq 2 g-1$, the space $E_{i, \ldots, i}$ is a manifold of dimension $2(n+1) i-2 n g=2(i-g)(n+1)+2 g$. However when $i<2 g-1$ there is no garantee that $E_{i, \ldots, i}$ is actually a manifold.

Using Alexander-Poincaré duality, we can now deduce

Lemma 2.8. Assume $i \geq 2 g-1$. Then for untwisted coefficients A we have

$$
H_{j}\left(H_{o l}^{*}\left(M_{g}, \mathbf{P}^{n}\right) ; \mathbf{A}\right) \cong H^{2(i-g)(n+1)+2 g-j}\left(Q E_{i} ; \mathbf{A}\right) .
$$


Note at this point that the multiplication $\mu$ of (2.3) induces an associative, commutative multiplication on the $Q E_{i}$ as well:

$$
\mu: Q E_{i} \times Q E_{j} \longrightarrow Q E_{i+j} .
$$

From Lemma (2.8), it is clear that it is the space $Q E_{i}$ that we wish to study in the remainder of this paper. Unfortunately, it is generally very hard to obtain the cohomology of such a space without a careful analysis of the piece we collapse out. So, in order to do this we follow the procedure of [5] and replace the cone on the union above by a more complex but much more structured space.

\section{A model for $Q E_{i}$}

Consider the twisted product space

$$
D E\left(M_{g}\right)=\left(\bigcup E_{i_{0}, i_{1}, \ldots, i_{n}}\right) \times_{t} S P^{\infty}\left(c M_{g}\right),
$$

where $c T$ denotes the reduced cone on $T$, and the twisting $t$ is given by the action above. Precisely, points of $D E$ are of the form

$$
\left.\left\{\left(D_{0}, \ldots, D_{n}\right),\left(t_{1}, z_{1}\right) \ldots\left(t_{l}, z_{l}\right)\right\}, D_{i} \in S P^{k_{i}}\left(M_{g}\right) \text { and } \mu\left(D_{i}\right)=\mu\left(D_{j}\right)\right\}
$$

with the identification that when $t_{i}=0$ the point above is identified with

$$
\left\{\left(D_{0}+z_{i}, \ldots, D_{n}+z_{i}\right),\left(t_{1}, z_{1}\right) \ldots \widehat{\left(t_{i}, z_{i}\right)} \ldots\left(t_{l}, z_{l}\right)\right\},
$$

where the entry $\left(t_{i}, z_{i}\right)$ is deleted from the last set of coordinates. Clearly $\mu\left(D_{r}+z_{i}\right)=\mu\left(D_{s}+z_{i}\right)$ and the construction makes sense.

The space $D E$ is filtered by the subspaces

$$
D E_{k_{0}, k_{1}, \ldots, k_{n}}\left(M_{g}\right)=\bigcup_{\substack{i_{0}+l \leq k_{0} \\ \vdots \\ i_{n}+i^{\prime} \leq k_{n}}} E_{i_{0}, i_{1}, \ldots, i_{n}} \times_{t} S P^{l}\left(c M_{g}\right) .
$$

Observe that there are projection maps

$$
p_{k_{0}, k_{1}, \ldots, k_{n}}: D E_{k_{0}, k_{1}, \ldots, k_{n}} \longrightarrow E_{k_{0}, k_{1}, \ldots, k_{n}} /\{\operatorname{Image}(\nu)\}
$$

where

$$
\left(v_{1}, \ldots, v_{s},\left(t_{1}, w_{1}\right), \ldots,\left(t_{r}, w_{r}\right)\right) \mapsto\left\{\left(v_{1}, \ldots, v_{s}\right)\right\}
$$


and the inverse images of points consist of contractible sets. This implies that the maps $p_{k_{0}, k_{1}, \ldots, k_{n}}$ are acyclic and induce isomorphisms

$$
H_{*}\left(D E_{k_{0}, k_{1}, \ldots, k_{n}} ; \mathbf{F}\right) \longrightarrow H_{*}\left(E_{k_{0}, k_{1}, \ldots, k_{n}} /\{\operatorname{Image}(\nu)\} ; \mathbf{F}\right) .
$$

We combine this with the isomorphism in Lemma 2.8 to get

Corollary 3.2. Let $k \geq 2 g-1$, then

$$
\begin{aligned}
& \tilde{H}^{2 k(n+1)-2 n g-*}\left(H_{o} l_{k}^{*}\left(M_{g}, \mathbf{P}^{n}\right) ; \mathbf{F}\right) \\
& \cong H_{*}(D E_{\underbrace{k, \ldots, k}_{n+1}} / \bigcup_{i} D E_{k, \ldots,}, \underbrace{k-1}_{i t h-\text { entry }}, \ldots, k ; \mathbf{F})
\end{aligned}
$$

This result is very useful because it is possible, using the filtration of the space $D E_{k, \ldots, k}$ described above, to construct spectral sequences with known $E_{1}$-terms which converge to the cohomology of the relative spaces above for all $k \geq 1$.

\section{The spectral sequence}

The diagonal action. The diagonal multiplication introduced in (2.3)

$$
S P^{r}\left(M_{g}\right) \times E_{i_{0}, i_{2}, \ldots, i_{n}} \stackrel{\nu}{\longrightarrow} E_{i_{0}+r, \ldots, i_{n}+r}
$$

induces an action in homology, $\nu_{*}$,

$$
\begin{gathered}
\nu_{*}:\left(\coprod_{r=0}^{\infty} H_{*}\left(S P^{r}\left(M_{g}\right) ; \mathbf{F}\right)\right) \otimes\left(\coprod_{j=1}^{\infty} H_{*}\left(L E_{i} ; \mathbf{F}\right)\right) \\
\longrightarrow \coprod_{j=1}^{\infty} H_{*}\left(L E_{i+r} ; \mathbf{F}\right)
\end{gathered}
$$

for any field coefficients F. Of course, this quotient action fits together with the original action on the $E_{i, \ldots, i}$ via the following commutative diagram

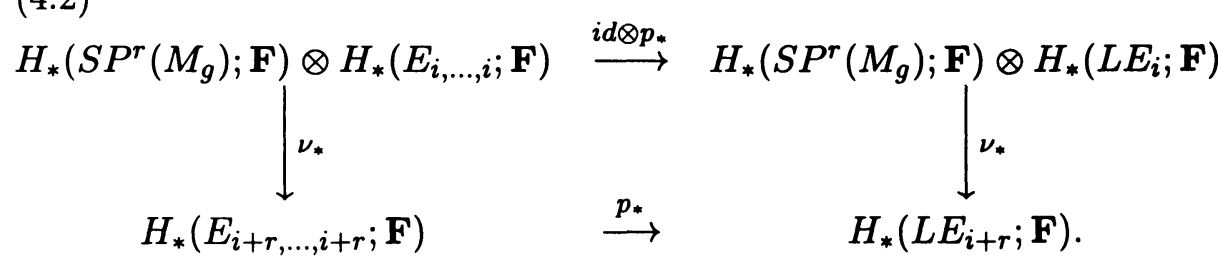


Theorem 4.3. There is a spectral sequence converging to $H_{*}\left(Q E_{k} ; \mathbf{F}\right)$ with $E^{1}$ term

$$
\begin{gathered}
E^{1}=\coprod_{\substack{i+j=k \\
i \geq 1}} \tilde{H}_{*}\left(L E_{i} ; \mathbf{F}\right) \otimes H_{*}\left(S P^{j}\left(\Sigma M_{g}\right), S P^{j-1}\left(\Sigma M_{g}\right) ; \mathbf{F}\right) \\
\oplus H_{*}\left(S P^{k}\left(\Sigma M_{g}\right), S P^{k-1}\left(\Sigma M_{g}\right) ; \mathbf{F}\right)
\end{gathered}
$$

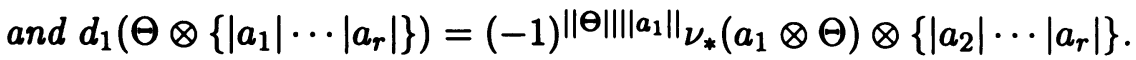

Proof. From the model for $Q E$ constructed in $\S 3$ we have that

$$
Q E_{k} \cong D E_{k, \ldots, k} / \bigcup D E_{k, \ldots, k-1, \ldots, k}
$$

where

$$
D E_{k, \ldots, k}\left(M_{g}\right)=\bigcup_{i_{r}+j \leq k} E_{i_{0}, i_{1}, \ldots, i_{n}} \times_{t} S P^{j}\left(c M_{g}\right) .
$$

Also, $L E_{i}=E_{i, \ldots, i} /\left\{\bigcup E_{i, i, \ldots, i-1, i, \ldots, i}\right\}$ so we can write

$$
Q E_{k}=\bigcup_{i+j=k} L E_{i} \times_{t}\left(S P^{j}\left(c M_{g}\right) / S P^{j-1}\left(c M_{g}\right)\right),
$$

where the twisting in the description above is given as before by

$$
\left(h,\left\{\left(0, w_{1}\right),\left(t_{2}, w_{2}\right), \ldots,\left(t_{r}, w_{r}\right)\right\}\right) \sim\left(\nu\left(w_{1}, h\right),\left\{\left(t_{2}, w_{2}\right), \ldots,\left(t_{r}, w_{r}\right)\right\}\right) .
$$

To obtain the desired spectral sequence, introduce the filtration by

$$
\mathcal{F}_{r}\left(Q E_{k}\right)=\bigcup_{\substack{i \leq r \\ i+j=k}} L E_{i} \times_{t}\left(S P^{j}\left(c M_{g}\right) / S P^{j-1}\left(c M_{g}\right)\right),
$$

and the remainder of the proof of the theorem is direct. q.e.d.

$A$ multiplicative structure for the spectral sequence. The induced multiplication on the $Q E_{i}$ 's,

$$
\bar{\nu}: Q E_{i} \times Q E_{j} \longrightarrow Q E_{i+j}
$$

passes to the spectral sequences above and defines a pairing of $E^{1}$-terms:

$$
\begin{aligned}
& \left(H_{*}\left(L E_{i} ; \mathbf{F}\right) \otimes H_{*}\left(S P^{j}\left(\Sigma M_{g}\right), S P^{j-1}\left(\Sigma M_{g}\right) ; \mathbf{F}\right)\right) \\
& \quad \otimes\left(H_{*}\left(L E_{v} ; \mathbf{F}\right) \otimes H_{*}\left(S P^{w}\left(\Sigma M_{g}\right), S P^{w-1}\left(\Sigma M_{g}\right) ; \mathbf{F}\right)\right) \\
& \quad \longrightarrow H_{*}\left(L E_{i+v} ; \mathbf{F}\right) \otimes H_{*}\left(S P^{w+j}\left(\Sigma M_{g}\right), S P^{w+j-1}\left(\Sigma M_{g}\right) ; \mathbf{F}\right)
\end{aligned}
$$


for which the $d_{i}$ 's act as derivations. For this reason it is often convenient to consider all the spectral sequences above at once. In particular we can describe the direct sum of all the $E_{1}$-terms as a trigraded ring where an element $x \in E_{1}$ has tridegree $(i, j, *)$ if and only if

$$
x \in H_{s}\left(L E_{i}\right) \otimes H_{*-s}\left(S P^{j}(\Sigma M), S P^{j-1}(\Sigma M) ; \mathbf{F}\right) .
$$

Remark 4.4. There is a related spectral sequence for $H_{*}\left(Q E_{k} ; \mathbf{F}\right)$ obtained by filtering

$$
Q E_{k}=\bigcup_{i+j=k} L E_{i} \times_{t}\left(S P^{j}\left(c M_{g}\right) / S P^{j-1}\left(c M_{g}\right)\right)
$$

in a somewhat different way. Instead of filtering by $i$ in the expression above, filter by the number of distinct $t$ 's in the point

$$
\left(h,\left\{\left(t_{1}, w_{1}\right),\left(t_{2}, w_{2}\right), \ldots,\left(t_{r}, w_{r}\right)\right\}\right) .
$$

In the space $S P^{\infty}\left(\Sigma M_{g}\right)$ this filtration results in the Eilenberg-Moore spectral sequence with $E_{2}$-term $\operatorname{Ext}_{H_{*}\left(S P^{\infty}\left(M_{g}\right) ; \mathbf{F}\right)}^{* *}(\mathbf{F}, \mathbf{F})$. To describe the resulting spectral sequence most efficiently it is best to include all the $Q E_{k}$ 's at once, and what results in our case is a trigraded $E_{2}$-term,

$$
\operatorname{Ext}_{H_{*, *}\left(S P^{\infty}\left(M_{g}\right) ; \mathbf{F}\right)}^{r, s, *}\left(\mathbf{F}, \bigoplus_{i=1}^{\infty} H_{*}\left(L E_{i} ; \mathbf{F}\right)\right),
$$

where the summand such that $r+s=k$ corresponds to the $E_{2}$-term of the spectral sequence converging to $H^{*}\left(Q E_{k} ; \mathbf{F}\right)$.

Some remarks on differentials. There are similar spectral sequences for the Div-spaces (cf. 1.6) starting (as in 3.1) with the model

$$
(\coprod_{j=0}^{\infty} \underbrace{S P^{j}\left(M_{g}\right) \times \cdots \times S P^{j}\left(M_{g}\right)}_{n+1 \text {-times }}) \times_{T} S P^{\infty}\left(c M_{g}\right),
$$

where $T$ is now the diagonal twisting which identifies the point $(0, z)$ in the cone $c M$ with the diagonal element $\Delta^{n+1}(z)$ in $\left(M_{g}\right)^{n+1}$ and then extends this multiplicatively. The associated spectral sequences have $E_{1}$-term

$$
\begin{aligned}
\coprod_{i+j=k}\left[H^{*}\left(S P^{i}\left(M_{g}\right), S P^{i-1}\left(M_{g}\right) ; \mathbf{F}\right)\right]^{n+1} \\
\otimes H^{*}\left(S P^{j}\left(\Sigma M_{g}\right), S P^{j-1}\left(\Sigma M_{g}\right) ; \mathbf{F}\right)
\end{aligned}
$$


and converge to $H^{*}\left(\operatorname{Div}_{k, \ldots, k}\left(M_{g}\right) ; \mathbf{F}\right)$. (Here the superscript $n+1$ means the $(n+1)$-fold tensor product.) Moreover, as is clear, the inclusions

$$
E_{i, \ldots, i} \subset S P^{i}\left(M_{g}\right) \times \cdots \times S P^{i}\left(M_{g}\right)
$$

induce maps of spectral sequences here (in cohomology) to the spectral sequences above for the $\mathrm{Hol}_{k}^{*}$ spaces. (Or in homology from the spectral sequences for the $\mathrm{Hol}_{k}^{*}$ spaces to these for the Div-spaces.)

But for the spectral sequences for the Div-spaces, and using H. Cartan's little constructions [4] to embed the homology into the chain complex one is able to construct an explicit (small) filtered chain complex with associated spectral sequence equal to that in Theorem 4.3, [12]. In particular one knows that $E_{\infty}=E_{1}$ for $n \geq 2$ for the Div-spaces spectral sequence 4.6.

In the next section we will identify a region of the spectral sequence for the $\mathrm{Hol}_{k}^{*}$ spaces where the induced map of homology spectral sequences is an injection. Hence, in this range for $n>1$ the spectral sequence for $\operatorname{Hol}_{k}^{*}\left(M_{g}, \mathbf{P}^{n}\right)$ collapses at $E_{1}$. When $n=1$ there are differentials, however our knowledge of the differentials in the Div-spectral sequence here implies considerable information about the differentials for the $\mathrm{Hol}_{k}^{*}$ spaces here as well.

The $d^{1}$-differential for the highest filtration terms of the spectral sequence. One region where the two sequences don't compare very well is the tail end of Theorem 4.3, the terms

$$
\tilde{H}_{*}\left(M_{g} ; \mathbf{F}\right) \otimes H_{*}\left(S P^{k-1}\left(\Sigma M_{g}\right), S P^{k-2}\left(\Sigma M_{g}\right) ; \mathbf{F}\right)
$$

and

$$
H_{*}\left(S P^{k}\left(\Sigma M_{g}\right), S P^{k-1}\left(\Sigma M_{g}\right) ; \mathbf{F}\right) .
$$

More generally it can happen that $\mu_{k}: S P^{k}\left(M_{g}\right) \rightarrow J\left(M_{g}\right)$ is an embedding for $1 \leq k \leq m\left(M_{g}\right)$ in which case we have

Lemma 4.7. If the Abel-Jacobi map $\mu_{k}: S P^{k}\left(M_{g}\right) \rightarrow J\left(M_{g}\right)$ is an embedding for $1 \leq k \leq m\left(M_{g}\right)$, then in this range we have $E_{k, \ldots, k} \cong$ $S P^{k}\left(M_{g}\right), E_{k, \ldots, k-1, k, \ldots, k} \cong S P^{k-1}\left(M_{g}\right)$ included in $E_{k, \ldots, k}$ via the usual base-point embedding

$$
S P^{k-1}(X) \subset S P^{k}(X), \quad\left\langle x_{1}, \ldots, x_{k-1}\right\rangle \mapsto\left\langle x_{1}, \ldots, x_{k-1}, *\right\rangle
$$

and the action $\nu$ corresponds to the usual multiplication

$$
S P^{r}\left(M_{g}\right) \times S P^{k-r}\left(M_{g}\right) \longrightarrow S P^{k}\left(M_{g}\right) .
$$


Consequently, in this range we have $L E_{k, \ldots, k} \cong S P^{k}\left(M_{g}\right) / S P^{k-1}\left(M_{g}\right)$, $1 \leq k \leq m\left(M_{g}\right)$, and the spectral sequence in this region is the corresponding spectral sequence for the quasi-fibration

$$
S P^{\infty}\left(M_{g}\right) \rightarrow S P^{\infty}\left(c M_{g}\right) \rightarrow S P^{\infty}\left(\Sigma M_{g}\right) .
$$

(The only statement above which might need clarification is the last. But recall that the spectral sequence with field coefficients has $E_{2}$-term

$$
\bigoplus_{k, t} H^{*}\left(S P^{k}\left(M_{g}\right), S P^{k-1}\left(M_{g}\right) ; \mathbf{F}\right) \otimes H^{*}\left(S P^{t}\left(\Sigma M_{g}\right), S P^{t-1}\left(\Sigma M_{g}\right) ; \mathbf{F}\right)
$$

and all the differentials preserve the sum $k+t$. Moreover, in this region the chain embedding techniques of [12] are valid, so the two spectral sequences have the same (internal) differentials.)

For example, if $M_{g}$ is not hyperelliptic then $\mu_{2}: S P^{2}\left(M_{g}\right) \rightarrow J\left(M_{g}\right)$ is an embedding. Also the map $\mu_{3}: S P^{3}\left(M_{5}\right) \rightarrow J\left(M_{5}\right)$ is an embedding for most curves of genus 5 . (See the discussion in $[1$, chapter $V]$.)

\section{The Jacobi varieties $W_{i}^{j}$ and a spectral sequence for the $L E_{i}$ spaces}

Our next step is to analyze the spectral sequence of Theorem 4.3. To do this we must understand the groups $H_{*}\left(L E_{i}\right)$. The $L E_{i}$ are quotients of the spaces $E_{i} \equiv E_{i, \ldots, i}$ defined in $\S 2$ and these latter spaces turn out to be built out of fibrations with projective spaces as fibers. Here there is a stratification of the image of $S P^{n}\left(M_{g}\right)$ in $J\left(M_{g}\right)$, and over each stratum we get such a fibration, though the dimensions of the fibers vary as we move from stratum to stratum.

Definition 5.1. The image of $\mu_{d}$ in $J\left(M_{g}\right)$ is written $W_{d}$. Also the set of points $y \in W_{d}$ so that $\mu_{d}^{-1}(y)=\mathbf{P}^{m}$ with $m \geq r$ is denoted $W_{d}^{r}$. Thus we have a decreasing filtration

$$
W_{d} \supseteq W_{d}^{1} \supseteq W_{d}^{2} \supseteq \cdots \supseteq W_{d}^{r} \supseteq \cdots \supseteq W_{d}^{[d / 2]+1}=\emptyset .
$$

It is well known that $W_{d}=J\left(M_{g}\right)$ whenever $d \geq g$, and that the dimension $m$ of a generic fiber $\mathbf{P}^{m}$ over $W_{d}$ is 0 when $d \leq g$ and $d-g$ when $d \geq g$. 


\section{Examples.}

(5.2) The map $\mu_{1}$ is always an embedding and so $W_{1} \cong M_{g}$.

(5.3) In the genus 1 case the original map $\mu=\mu_{1}$ is the identity, and $J\left(M_{1}\right)=M_{1}$, while the $\mu_{d}$ are fiberings for $d \geq 2$.

Assume now that $g \geq 2$.

(5.4) The map $\mu_{2}: S P^{2}\left(M_{g}\right) \rightarrow J\left(M_{g}\right)$ is an embedding unless $M_{g}$ is hyperelliptic (cf $\S 10)$. In the hyperelliptic case $W_{2}^{1}$ is a single point $p$ and $\mu_{2}^{-1}(p)=\mathbf{P}^{1}$. It follows that $W_{2}$ can be identified with $S P^{2}\left(M_{g}\right)$ with a single $\mathbf{P}^{1}$ blown down. Indeed, we can check that the normal bundle to $\mathbf{P}^{1} \subset S P^{2}\left(M_{g}\right)$ is $\xi^{1-g}$, the line bundle with self-interesection number $1-g$, and from this it follows that

(1) $S P^{2}\left(M_{2}\right)$ is the blowup of $J\left(M_{2}\right)$ at a single point (here any genus 2 surface is automatically hyperelliptic).

(2) For $g \geq 3$ we have that $W_{2}=S P^{2}\left(M_{g}\right) /\left(\mathbf{P}^{1} \sim *\right)$ is a manifold with a single isolated point singularity which looks like the cone on a Lens space $L_{g-1}^{3}$.

We can introduce the complementary subspace $A_{k}^{i}=W_{k}^{i}-W_{k}^{i+1}$. By definition, we have that $\forall x \in A_{k}^{i}, \mu_{k}^{-1}(x)=\mathbf{P}^{i}$. The result of Mattuck quoted in (1.14) takes actually the more general form.

Theorem 5.5 (Mattuck). $\mu_{k}^{-1}\left(A_{k}^{i}\right) \subset S P^{k}\left(M_{g}\right)$ is the total space of a locally trivial analytic fibration $\mathbf{P}^{i} \rightarrow \mu_{k}^{-1}\left(A_{k}^{i}\right) \rightarrow A_{k}^{i}$.

Remark 5.6. The following formula relating $W_{k}^{i}$ and $W_{k-1}^{i-1}$ is a special case of $[10,(20)$, p. 53$]$ :

$$
W_{k}^{i}=\bigcap_{m \in \mu\left(M_{g}\right)}\left(W_{k-1}^{i-1}+m\right),
$$

that is, $W_{k}^{i}$ is obtained as the intersection of all translates of elements of $W_{k-1}^{i-1}$ by elements of $\mu\left(M_{g}\right) \subset J\left(M_{g}\right)$. In particular, this shows that $(* * *)$

$$
W_{k}^{i} \subset W_{k-1}^{i-1}+* \subset W_{k}^{i-1} \text {. }
$$

From now on we do not differentiate between $W_{k-1}^{i-1} \subset W_{k-1}$ and $W_{k-1}^{i-1}+* \subset W_{k}$; denoting both by $W_{k-1}^{i-1}$.

Furthermore, Gunning, [10, p. 54], gives the following dimension estimates for the associated containments.

Lemma 5.7. If the subvariety $W_{k-1}^{i-1}$ is non-empty then: 
- $\operatorname{dim} W_{k}^{i}<\operatorname{dim} W_{k-1}^{i-1}$ whenever $2 \leq i \leq k \leq g$.

- $\operatorname{dim} W_{k-2}^{i-1}<\operatorname{dim} W_{k-1}^{i-1}$ whenever $2 \leq i \leq k \leq g$.

Remark 5.8. In chapter $\mathrm{V}$ of [1] techniques for determining the $W_{k}^{i}$, s are extensively discussed, and results for $g \leq 6$ are completely given, (p. 206-211).

We now turn to the pull-back spaces $E_{k, \ldots, k}$ and observe that the fibration of Lemma (5.5) induces in turn a fibration

$$
\left(\mathbf{P}^{i} \times \cdots \times \mathbf{P}^{i}\right) \longrightarrow \mathcal{A}_{k}^{i} \stackrel{\pi}{\longrightarrow} A_{k}^{i}, \mathcal{A}_{k}^{i} \subset E_{k, \ldots, k} .
$$

When collapsing $\mathcal{A}_{k-1}^{i}$, we get a quotient

$$
X=\mathcal{A}_{k}^{i} / \mathcal{A}_{k-1}^{i} \hookrightarrow E_{k, \ldots, k} / E_{k-1, \ldots, k-1}
$$

and a quotient map $X \longrightarrow L E_{k, \ldots, k}$. The space $X$ projects down via $\mu$ to

$$
A_{k}^{i} / A_{k-1}^{i}=W_{k}^{i} / W_{k-1}^{i} ;
$$

this last equality being a consequence of the inclusion $W_{k}^{i+1} \subset W_{k-1}^{i}$ described above.

We then pass to the quotient $L E_{k}$ and observe that since $W_{k}^{i} \subset W_{k-1}^{i-1}, i \geq 1$, we must further collape, along fibers this time, subsets of the form

$$
\bigcup_{j \leq n+1}\left(\mathbf{P}^{i}\right)^{j-1} \times \mathbf{P}^{i-1} \times\left(\mathbf{P}^{i}\right)^{n+1-j} \hookrightarrow\left(\mathbf{P}^{i}\right)^{n+1} .
$$

The fiber $\left(\mathbf{P}^{i}\right)^{n+1}$ has a top $2 i(n+1)$ cell of the form

$$
e^{2 i(n+1)}=e_{1}^{2 i} \times \cdots \times e_{n+1}^{2 i},
$$

where $e_{j}^{2 i}$ is the top $2 i$ dimensional cell in the $j$-th copy $\mathbf{P}^{i}$ with boundary mapping to $\mathbf{P}^{i-1} \subset \mathbf{P}^{i}$. We then see that

$$
\left(\mathbf{P}^{i}\right)^{n+1} / \cup_{j}\left(\mathbf{P}^{i}\right)^{j-1} \times \mathbf{P}^{i-1} \times\left(\mathbf{P}^{i}\right)^{n+1-j} \simeq e^{2 i(n+1)} / \partial e^{2 i(n+1)} \simeq S^{2 i(n+1)} .
$$

The space so obtained is denoted by $T_{k}^{i}$. In the case when $i=0$ we have that $W_{k}^{1} \subset W_{k-1} \subset W_{k}$, and hence $W_{k} / W_{k-1} \cong S P^{k}\left(M_{g}\right) / S P^{k-1}\left(M_{g}\right)$.

Now we filter the Jacobian according to the increasing sequence of $W_{i}^{\prime}$ s

$$
J\left(M_{g}\right)=W_{g} \supset W_{g-1} \supset \cdots \supset W_{1}=M_{g} .
$$


This induces a filtration on $L E_{k}$ yielding a spectral sequence which by the preceeding discussion has $E^{1}$ term as follows.

Proposition 5.9. Suppose that $k \leq g$. Then there is a spectral sequence converging to $\tilde{H}_{*}\left(L E_{k}\right)$ with $E^{1}$ term

$$
E^{1}=H_{*}\left(S P^{k}\left(M_{g}\right), S P^{k-1}\left(M_{g}\right)\right) \oplus \coprod_{i} H_{*}\left(W_{k}^{i}, W_{k-1}^{i}\right) \otimes \tilde{H}_{*}\left(S^{2 i(n+1)}\right)
$$

and in case $k \geq g$, then

$$
\begin{aligned}
& E^{1}=\Sigma^{2(k-g)(n+1)} H_{*}\left(J\left(M_{g}\right), W_{k-1}^{k-g}\right) \\
& \oplus \coprod_{i>k-g} H_{*}\left(W_{k}^{i}, W_{k-1}^{i}\right) \otimes \tilde{H}_{*}\left(S^{2 i(n+1)}\right) .
\end{aligned}
$$

Remark 5.10. Note that

$$
H_{*}\left(L E_{k}\right)=H_{*}\left(J\left(M_{g}\right)\right) \otimes \tilde{H}_{*}\left(S^{2(k-g)(n+1)}\right),
$$

whenever $k>2 g-1$ for then $W_{k-1}^{k-g}=\emptyset$ and $W_{k}^{i}=W_{k-1}^{i}=J\left(M_{g}\right)$ otherwise.

Remark 5.11. The spectral sequence of (5.9) turns out to collapse at $E^{1}$ for all cases that we treat in this paper.

\section{The structure of symmetric products}

In this section, we describe the homology of the symmetric products $S P^{n}\left(M_{g}\right)$ for all $n$ and $g$. Also, since it is required in the spectral sequences of Theorem 4.3 and Proposition 5.9, we give the structure of $H_{*}\left(S P^{n}\left(\Sigma M_{g}\right)\right)$ again for all $n, g$. In the case of $S P^{n}\left(M_{g}\right)$ we follow the description given by I.G. Macdonald, [15], while the description for the suspension is taken from [19] and unpublished work of N. Steenrod.

There is an evident pairing $S P^{n}(X) \times S P^{m}(X) \stackrel{+}{\rightarrow} S P^{n+m}(X)$ given by addition of points, and this turns $S P^{\infty}(X)$ into an associative, abelian monoid with $*$ as a two sided identity. The Dold and Thom theorem states that

$$
S P^{\infty}(X) \simeq \prod_{1}^{\infty} K\left(H_{i}(X ; \mathbf{Z}), i\right)
$$


is a product of Eilenberg-MacLane spaces if $X$ is path connected [7]. Applying this to $X=M_{g}$ yields

$$
S P^{\infty}\left(M_{g}\right) \simeq K\left(\mathbf{Z}^{2 g}, 1\right) \times K(\mathbf{Z}, 2) \simeq\left(S^{1}\right)^{2 g} \times \mathbf{P}^{\infty},
$$

where $K(\mathbf{Z}, 1) \simeq S^{1}$ while $K(\mathbf{Z}, 2) \simeq \mathbf{P}^{\infty}$, the infinite complex projective space. We then find that

$$
H_{*}\left(S P^{\infty}\left(M_{g}\right) ; \mathbf{Z}\right) \cong \Lambda\left(e_{1}, \ldots, e_{2 g}\right) \otimes \Gamma(a),
$$

where $\Lambda($,$) denotes the exterior algebra on the stated generators while$ $\Gamma(a)$ denotes the divided power algebra on $a$ : the ring with $\mathbf{Z}$-generators $a_{i}, \quad i=0,1, \ldots$ and multiplication $a_{i} a_{j}=\left(\begin{array}{c}i+j \\ j\end{array}\right) a_{i+j}$. This accords well with the multiplication of the $S P^{n}\left(M_{g}\right)$ described above. Often however it is convenient to work with the cohomology rings so we need the following description.

I.G. Macdonald's description of $H^{*}\left(S P^{k}\left(M_{g}\right) ; \mathbf{Z}\right)$.

Consider the map $\left[M_{g}\right]^{*}: M_{g} \rightarrow \mathbf{P}^{\infty}=K(\mathbf{Z}, 2)$, taking the fundamental class to the dual of the orientation class, and the map

$$
\vee e_{i}: M_{g} \rightarrow K\left(\mathbf{Z}^{2 g}, 1\right) \simeq\left(S^{1}\right)^{2 g} .
$$

Both $\mathbf{P}^{\infty}$ and $\left(S^{1}\right)^{2 g}$ are associative abelian $H$-spaces with the structure on $\mathbf{P}^{\infty}$ coming from the identification $S P^{\infty}\left(\mathbf{P}^{1}\right)=\mathbf{P}^{\infty}$ described earlier.

This allows us to extend $\left[M_{g}\right]^{*}$ and $\vee e_{i}$ to a multiplicative map

$$
\theta(k):\left(M_{g}\right)^{k} \rightarrow \mathbf{P}^{\infty} \times\left(S^{1}\right)^{2 g}
$$

which, by definition factors through the symmetric product $S P^{k}\left(M_{g}\right)$. In the limit, as $k \mapsto \infty$ this gives the Dold-Thom equivalence

$$
S P^{\infty}\left(M_{g}\right) \rightarrow K\left((\mathbf{Z})^{2 g}, 1\right) \times K(\mathbf{Z}, 2) .
$$

With respect to the maps $\theta(k)$ we can describe the cohomology ring $H^{*}\left(S P^{k}\left(M_{g}\right) ; \mathbf{Z}\right)$ as follows:

Theorem 6.4 (I.G. Macdonald). The cohomology ring of $S P^{k}\left(M_{g}\right)$ over the integers $\mathbf{Z}$ is generated by the elements

$$
f_{1}=e_{1}^{*}, \ldots, f_{i}=e_{2 i-1}^{*}, \ldots f_{g}=e_{2 g-1}^{*}, f_{1}^{\prime}=e_{2}^{*}, \ldots f_{g}^{\prime}=e_{2 g}^{*}, \text { and } b
$$

subject to the following relations: 
(i) The $f_{i}$ 's and the $f_{i}^{\prime}$ 's anti-commute with each other and commute with $b$;

(ii) If $i_{1}, \ldots, i_{a}, j_{1}, \ldots, j_{b}, k_{1}, \ldots, k_{c}$ are distinct integers from 1 to $g$ inclusive, then

$$
f_{i_{1}} \cdots f_{i_{a}} f_{j_{1}}^{\prime} \cdots f_{j_{b}}^{\prime}\left(f_{k_{1}} f_{k_{1}}^{\prime}-b\right) \cdots\left(f_{k_{c}} f_{k_{c}}^{\prime}-b\right) b^{q}=0
$$

provided that

$$
a+b+2 c+q=n+1 .
$$

If $k<2 g$ all the relations above are consequences of those for which $q=0$, and if $n>2 g-2$ all the relations are consequences of the single relation

$$
b^{k-2 g+1} \prod_{i=1}^{g}\left(f_{i} f_{i}^{\prime}-b\right)=0 .
$$

(Actually Macdonald only stated this result in [15] for fields of characteristic zero as coefficients, however, since the relevant invariant maps that he used to prove the result are actually surjective over $\mathbf{Z}$, it is quite direct to extend the result to integers as well.)

The homology of $S P^{n}(X)$ for more general $X$

The homology of the spaces $S P^{n}(X)$ splits according to a result of Steenrod, [6], (see also [19]) which holds for $X$ any $C W$-complex:

$$
H_{*}\left(S P^{\infty}(X) ; \mathbf{Z}\right)=\bigoplus_{j} H_{*}\left(S P^{j}(X), S P^{j-1}(X) ; \mathbf{Z}\right) .
$$

Consequently, the ring $H_{*}\left(S P^{\infty}\left(M_{g}\right) ; \mathbf{Z}\right)$ is bigraded, by defining $x \in$ $H_{*}\left(S P^{\infty}(X) ; \mathbf{Z}\right)$ to have bidegree $(i, j)$ iff

$$
x \in H_{j}\left(S P^{i}\left(M_{g}\right), S P^{i-1}\left(M_{g}\right) ; \mathbf{Z}\right) \subset H_{*}\left(S P^{\infty}\left(M_{g}\right) ; \mathbf{Z}\right) .
$$

The bigrading is multiplicative in the sense that the product map

$$
S P^{m}(X) \times S P^{n}(X) \rightarrow S P^{n+m}(X)
$$

in homology induces a bigraded ring map

$$
H_{i, j}\left(S P^{n}(X) ; \mathbf{A}\right) \otimes H_{r, s}\left(S P^{m}(X) ; \mathbf{A}\right) \longrightarrow H_{i+r, j+s}\left(S P^{n+m}(X) ; \mathbf{A}\right),
$$

where the first degree is the dimension and the second the bidegree. 
The bigrading is preserved by the diagonal map, so the cohomology ring is bigraded as well, and the two structures together form a bigraded Hopf algebra. In the case of the $S P^{k}\left(M_{g}\right)$ we have that the coproduct on each of the 1-dimensional generators is primitive, while the 2-dimensional generator $\left[M_{g}\right]$ in $H_{1,2}\left(S P^{\infty}\left(M_{g}\right) ; \mathbf{Z}\right)$ corresponding to the orientation class of $M_{g}$ has coproduct

$$
\Delta\left(\left[M_{g}\right]\right)=\left[M_{g}\right] \otimes 1+\sum_{1}^{g}\left(e_{2 i-1} \otimes e_{2 i}-e_{2 i} \otimes e_{2 i-1}\right)+1 \otimes\left[M_{g}\right]
$$

The homology of $S P^{n}\left(\Sigma\left(M_{g}\right)\right)$.

We will need the cohomology and homology of the spaces $S P^{n}\left(\Sigma\left(M_{g}\right)\right)$ where $\Sigma X$ denotes the suspension of $X$. From the DoldThom Theorem we have

$$
S P^{\infty}\left(\Sigma M_{g}\right) \simeq\left(\prod_{1}^{2 g} \mathbf{P}^{\infty}\right) \times S P^{\infty}\left(S^{3}\right)
$$

where we can assume that the generator for the homology of each $\mathbf{P}^{\infty}$ has bidegree $(1,2)$. Thus, this amounts to describing the homology and cohomology of $K(\mathbf{Z}, 3)$ and its associated bigrading.

With coefficients $\mathbf{F}_{p}$ for any odd prime $p$ we have, [19],

$$
\begin{aligned}
& H_{*}\left(K(\mathbf{Z}, 3) ; \mathbf{F}_{p}\right) \\
& \cong \Lambda\left(|a|,\left|\gamma_{p}\right|, \cdots\left|\gamma_{p^{i}}\right| \cdots\right) \otimes \Gamma\left(\left|a^{p-1}\right| a \mid\right) \\
& \quad \otimes \Gamma\left(\left|\gamma_{p}^{p-1}\right| \gamma_{p} \mid\right) \otimes \cdots \otimes \Gamma\left(\left|\gamma_{p^{i}}^{p-1}\right| \gamma_{p^{i}} \mid\right) \otimes \cdots .
\end{aligned}
$$

The generator $\gamma_{p^{i}}$ corresponds to $\left[S P^{p^{i}}\left(M_{g}\right)\right]$ and $\left|\gamma_{p^{i}}\right|$ has filtration degree $p^{i}$ and dimension $2 p^{i}+1$ while $\left|\gamma_{p^{i-1}}^{p-1}\right| \gamma_{p^{i-1}} \mid$ has bidegree $\left(p^{i}, 2 p^{i}+2\right)$.

In the case of the prime 2 we have

$$
H_{*}\left(K(\mathbf{Z}, 3) ; \mathbf{F}_{2}\right) \cong \Gamma\left[|a|,\left|a_{2}\right|, \ldots,\left|a_{2^{i}}\right|, \ldots\right] .
$$

Remark 6.10. With $F_{p}$ coefficients, the divided power algebra $\Gamma[a]$ splits as an algebra into a tensor product of truncated polynomial algebras [4]

$$
\Gamma[a] \cong \mathbf{F}_{p}[a] / a^{p} \otimes \mathbf{F}_{p}\left[a_{p}\right] /\left(a_{p}\right)^{p} \otimes \cdots
$$


When $\mathbf{F}=\mathbf{Q}$, divided power algebras are isomorphic to polynomial algebras and the answer simplifies greatly; namely

$$
H_{*}\left(S P^{\infty}\left(\Sigma M_{g}\right) ; \mathbf{Q}\right)=\mathbf{Q}\left[\left|e_{1}\right|, \ldots,\left|e_{2 g}\right|\right] \otimes \Lambda(a) .
$$

The answer is simpler to describe if we use cohomology. Here, $H^{*}\left(S P^{\infty}\left(S^{3}\right) ; \mathbf{F}_{p}\right)$ is always a tensor product of an exterior algebra on odd dimensional generators and a polynomial algebra on the even dimensional generators. In other words in the formula (6.8) above one replaces all the divided power algebras by polynomial algebras with generators of the same bidegrees to get the cohomology ring description.

We take advantage of the bigrading to write $H_{*}\left(S P^{\infty}\left(\Sigma M_{g}\right) ; \mathbf{F}_{p}\right)$ in the form

$$
\coprod_{1}^{\infty} H_{*}\left(S P^{n}\left(\Sigma M_{g}\right), S P^{n-1}\left(\Sigma M_{g}\right) ; \mathbf{F}_{p}\right) \text {. }
$$

These summands can be written out more completely in terms of the bigrading of $S P^{\infty}\left(S^{3}\right)$ as follows. First, we write $H_{*}\left(S P^{\infty}\left(S^{3}\right) ; \mathbf{F}_{p}\right)=$ $H_{*}\left(S P^{\infty}\left(\Sigma S^{2}\right) ; \mathbf{F}_{p}\right)$ in this way. The decomposition here has an alternate description, [5]:

$$
H_{*}\left(S P^{\infty}\left(S^{3}\right) ; \mathbf{F}_{p}\right)=\bigoplus_{1}^{\infty} \Sigma^{4 i} \mathcal{D}_{i}^{*}(p),
$$

where the $\mathcal{D}_{i}$ are the Snaith splitting components of the loop space $\Omega^{2} S^{3}$ for the prime $p$, and $\mathcal{D}_{i}^{*}$ means dual, where we index the dual by $\operatorname{dim}\left(\mathcal{D}_{i, j}\right)=-j$.

Then, going back to (6.7) we see that we can write the term

$$
\begin{aligned}
H_{*}\left(S P^{n}\left(\Sigma M_{g}\right), S P^{n-1}\left(\Sigma M_{g}\right) ; \mathbf{F}_{p}\right) \\
\quad=\bigoplus_{j=0}^{n} \Sigma^{4 j} \mathcal{D}_{j}^{*}(p) \otimes \mathbf{F}_{p}\left[b_{1}, \ldots, b_{2 g}\right]_{n-j}
\end{aligned}
$$

and $\mathbf{F}_{p}\left[b_{1}, \ldots, b_{2 g}\right]_{n-j}$ is the free $\mathbf{Z}$-module on the degree $n-j$ monomials in the variables $b_{1}, \ldots, b_{2 g}$. There are $\left(\begin{array}{c}n-j+2 g-1 \\ 2 g-1\end{array}\right)$ such monomials.

Example. What follows is a list of generators for

$$
H_{*}\left(S P^{j}(\Sigma M), S P^{j-1}(\Sigma M) ; \mathbf{Z}_{p}\right)
$$


in the $E^{1}$ term $\bmod (p)$ of the spectral sequence of Theorem 4.3 together with their tridegrees

$\begin{array}{cc}\text { Generator } & \text { trigrading } \\ |M| & (0,1,3) \\ \left|e_{i}\right| & (0,1,2) \\ \left|\gamma_{p^{i}}\right| & \left(0, p^{i}, 2 p^{i}+1\right) \\ \left|\gamma_{p^{i-1}}^{p-1}\right| \gamma_{p^{i-1}} \mid & \left(0, p^{i}, 2 p^{i}+2\right) .\end{array}$

Explicit $d^{1}$-differentials in the spectral sequence.

In Theorem 4.3 the differential $d^{1}$ is implicitly determined. Now that we have the explicit form of the homology groups $H_{*}\left(S P^{n}\left(M_{g}\right)\right)$ and $H_{*}\left(S P^{n}\left(\Sigma M_{g}\right)\right)$ we can make $d^{1}$ explicit. For example we have

$$
\begin{aligned}
d^{1} & \left(\left|f_{1}\right|^{s_{1}} \cdots\left|f_{2 g}\right|^{k-s_{1}-\cdots-s_{2 g-1}}\right) \\
& =\sum_{1}^{2 g} f_{i} \otimes\left|f_{1}\right|^{s_{1}} \cdots\left|f_{i}\right|^{s_{i}-1} \cdots\left|f_{2 g}\right|^{k-s_{1}-\cdots-s_{2 g-1}}
\end{aligned}
$$

and

$$
\begin{aligned}
& d^{1}\left(\left|M_{g}\right|\left|f_{1}\right|^{s_{1}} \cdots\left|f_{2 g}\right|^{k-s_{1}-\cdots-1}\right. \\
& \quad=\left[M_{g}\right] \otimes\left|f_{1}\right|^{s_{1}} \cdots\left|f_{2 g}\right|^{k-s_{1}-\cdots-1} \\
& \quad-\left(d^{1}\left(\left|f_{1}\right|^{s_{1}} \cdots\left|f_{2 g}\right|^{k-s_{1}-\cdots-1}\right)\left(1 \otimes\left|M_{g}\right|\right) .\right.
\end{aligned}
$$

It is easily checked that this part of the $d^{1}$-differential is injective to the term $\tilde{H}^{*}\left(M_{g} ; \mathbf{F}\right) \otimes \mathbf{F}\left[\left|f_{1}\right|, \ldots,\left|f_{2 g}\right|\right]_{k-1}$ with cokernel spanned by the monomials of the following form:

$$
\left\{f_{t}\left|f_{i}\right|^{j_{i}}\left|f_{i+1}\right|^{j_{i+1}} \cdots\left|f_{2 g}\right|^{k-1-j_{1}-\cdots-j_{2 g-1}}\right\},
$$

where $j_{i}>0$, and $t>i$.

Remark 6.18. This is the only time the $d^{1}$ differential on the elements $\left|f_{1}\right|^{i_{1}} \cdots\left|f_{2 g}\right|^{i_{2 g} g}$ is non-trivial, since in the remaining parts of the spectral sequence their images lie in the part which has been collapsed out.

Example 6.19. If $g=1$ then the differentials in this region have the form $d^{1}\left(\left|f_{1}\right|^{s}\left|f_{2}\right|^{k-s}\right)=f_{1} \otimes\left|f_{1}\right|^{s-1}\left|f_{2}\right|^{k-s}+f_{2} \otimes\left|f_{1}\right|^{s}\left|f_{2}\right|^{k-s-1}$ 
provided $s$ and $k-s$ are both greater than zero and

$$
\begin{aligned}
d^{1}\left(|T|\left|f_{1}\right|^{s}\left|f_{2}\right|^{k-s-1}\right)= & f_{1} f_{2} \otimes\left|f_{1}\right|^{s}\left|f_{2}\right|^{k-s-1} \\
& -f_{1} \otimes|T|\left|f_{1}\right|^{s-1}\left|f_{2}\right|^{k-s-1} \\
& -f_{2} \otimes|T|\left|f_{1}\right|^{s}\left|f_{2}\right|^{k-s-2} .
\end{aligned}
$$

The role of this last differential can be regarded as identifying terms of the form $f_{1} f_{2} \otimes\left|f_{1}\right|^{s}\left|f_{2}\right|^{k-s-1}$ with terms involving $|T|^{*}$. Also, the first of these differentials is injective but not surjective. The quotient has as a basis the set of images of the elements $f_{2} \otimes\left|f_{1}\right|^{s}\left|f_{2}\right|^{k-s-1}$ with $s>0$. Consequently it has dimension $k-2$.

\section{The stable range}

In the case where $i \geq 2 g$, we've seen already that the Abel-Jacobi map becomes an analytic fibration, and hence the projection

$$
E_{i, \ldots, i} \rightarrow J\left(M_{g}\right)
$$

fibers as

$$
\mathbf{P}^{i-g} \times \ldots \times \mathbf{P}^{i-g} \longrightarrow E_{i, \ldots, i} \longrightarrow J\left(M_{g}\right) .
$$

Because of this and other stabilization properties ( $\S 8)$, we refer to the range $i \geq 2 g$ as stable. In this case, we see that the relative groups

$$
\tilde{H}\left(L E_{i}, \mathbf{A}\right) \cong H_{*}\left(E_{i, i, \ldots, i}, \bigcup E_{i, \ldots, i-1, \ldots, i} ; \mathbf{A}\right)
$$

are given by

$$
\begin{array}{r}
{\left[H_{*}\left(\mathbf{P}^{i-g}, \mathbf{P}^{i-g-1} ; \mathbf{A}\right)\right]^{n+1} \otimes H_{*}\left(J\left(M_{g}\right) ; \mathbf{A}\right)} \\
\cong \Sigma^{2(i-g)(n+1)} H_{*}\left(J\left(M_{g}\right) ; \mathbf{A}\right) .
\end{array}
$$

Dually, in cohomology the relative group is given as

$$
\begin{aligned}
b_{0}^{i-g} b_{1}^{i-g} \cdots b_{n}^{i-g} \otimes H^{*}\left(J\left(M_{g}\right) ; \mathbf{A}\right) & \\
= & \left(b_{0} \cdots b_{n}\right)^{i-g} \otimes H^{*}\left(J\left(M_{g}\right) ; \mathbf{A}\right),
\end{aligned}
$$

a form which is often of more use in calculations. 
Remark 7.4. Of course, in both these forms, the inclusions $\mathbf{P}^{i-g} \subset S P^{i}\left(M_{g}\right)$ induce injections in homology onto Z-direct summands, but even more, from Macdonald's Theorem 6.4 it follows that the inclusion of pairs

$$
\left(\mathbf{P}^{i-g}, \mathbf{P}^{i-g-1}\right) \subset\left(S P^{i}\left(M_{g}\right), S P^{i-1}\left(M_{g}\right)\right)
$$

induces an inclusion in homology sending $H_{2(i-g)}\left(\mathbf{P}^{i-g}, \mathbf{P}^{i-g-1} ; \mathbf{Z}\right)$ isomorphically to the group $H_{2(i-g)}\left(S P^{i}\left(M_{g}\right), S P^{i-1}\left(M_{g}\right) ; \mathbf{Z}\right)=\mathbf{Z}$. (We will expore this fact further below.)

Thus in this stable range the $E_{1}$-term for $\operatorname{Hol}_{k}^{*}\left(M_{g}, \mathbf{P}^{n}\right)$ injects into the corresponding $E_{1}$-term for the $D i v$-space as was asserted at the end of $\S 4$. In particular this gives as a corollary to the results of [12],

Lemma 7.5. The spectral sequence of Theorem 4.3 collapses at $E_{1}$ in the stable range for $n \geq 2$.

Now we turn to some fairly direct calculations which lead to the determination of the $d^{1}$-differential in the $\mathrm{Hol}_{k}^{*}$ spectral sequence for $n=1$ in the stable range.

More exactly, in the stable range the relative group injects,

$$
H^{*}\left(E_{i, i}, E_{i, i-1} \cup E_{i-1, i} ; \mathbf{A}\right) \subset H^{*}\left(E_{i, i}, \mathbf{A}\right)
$$

as the principal ideal

$$
\left(\left(b_{0} b_{1}\right)^{i-2 g} W_{0} W_{1}\right) \subset H^{*}\left(E_{i, i} ; \mathbf{A}\right),
$$

where $W_{j}$ is the polynomial

$$
\begin{aligned}
W_{j} & =\prod_{t=1}^{g}\left(f_{2 t-1} f_{2 t}-b_{j}\right) \\
& =(-1)^{g}\left[b_{j}^{g}-\left(\sum_{1}^{g} f_{2 t-1} f_{2 t}\right) b_{j}^{g-1}+\cdots+(-1)^{g}\left(\prod_{1}^{2 g} f_{t}\right)\right],
\end{aligned}
$$

which generates the kernel of the restriction map to $H^{*}\left(E_{i-1, i} ; \mathbf{A}\right)$ and $H^{*}\left(E_{i, i-1} ; \mathbf{A}\right)$ respectively according to Macdonald's Theorem 6.4.

Lemma 7.8. In the stable range the differential $d_{1}$ in the spectral sequence of Theorem 4.3 vanishes for $n>1$. For $n=1$, it has the form

$$
d_{1}\left(W_{0} W_{1} b_{0}^{i-2 g} b_{1}^{i-2 g}\right)=2\left(\sum_{1}^{g} f_{2 i-1} f_{2 i}\right)\left(W_{0} W_{1} b_{0}^{i-2 g-1} b_{1}^{i-2 g-1}\right)\left|M_{g}^{*}\right|
$$


and it is multiplicative in the remaining generators. That is to say,

$$
\Theta W_{0} W_{1} b_{0}^{i-2 g} b_{1}^{i-2 g} \stackrel{d_{1}}{\longrightarrow} \pm \Theta d_{1}\left(W_{0} W_{1} b_{0}^{i-2 g} b_{1}^{i-2 g}\right) .
$$

Proof. We check the action map $M \times E_{i-1, \ldots, i-1} \rightarrow E_{i, \ldots, i}$ in cohomology. The term $f_{2 r-1} f_{2 r}-b_{i}$ maps to

$$
f_{2 r-1} \otimes f_{2 r}-f_{2 r} \otimes f_{2 r-1}+1 \otimes\left(f_{2 r-1} f_{2 r}-b_{i}\right),
$$

since $f_{2 r-1} f_{2 r}-b=0$ in $H^{2}\left(M_{g} ; \mathbf{Z}\right)$. Hence

$$
W_{i} \mapsto \sum_{r=1}^{g}\left(f_{2 r-1} \otimes f_{2 r}-f_{2 r} \otimes f_{2 r-1}\right) 1 \otimes W_{i}(r)+1 \otimes W_{i},
$$

where, as is evident $W_{i}(r)=\prod_{j \neq r}\left(f_{2 j-1} f_{2 j}-b_{i}\right)$. Thus, since $f_{i} f_{j}=0$ in $H^{*}\left(M_{g} ; \mathbf{Z}\right)$ for $\langle i, j\rangle \neq\langle 2 r-1,2 r\rangle$ for some $r$, it follows that

$$
\begin{aligned}
W_{0} W_{1} \mapsto & \sum_{r=1}^{g} 2 b \otimes\left(f_{2 r-1} f_{2 r} W_{0}(r) W_{1}(r)\right) \\
& +\left(f_{2 r-1} \otimes f_{2 r}-f_{2 r} \otimes f_{2 r-1}\right) 1 \otimes\left(W_{0}(r) W_{1}+W_{0} W_{1}(r)\right) \\
& +1 \otimes W_{0} W_{1} .
\end{aligned}
$$

Next note that $W_{i} b_{i}^{i-2 g}=0$ in $H^{*}\left(E_{i-1, \ldots, i-1} ; \mathbf{Z}\right)$, so the image of $\left(b_{0} b_{1}\right)^{i-2 g} W_{0} W_{1}$ is $2 b \otimes \sum f_{2 r-1} f_{2 r} b_{0}^{i-2 g-1} b_{1}^{i-2 g-1} W_{0} W_{1}$ since

$$
b_{i} W_{i}(r) f_{2 r-1} f_{2 r}=W_{i} f_{2 r-1} f_{2 r} .
$$

This proves the desired result. q.e.d.

Remark 7.9. Actually, what the argument above really determines is the coaction map in cohomology. Given the action map

$$
S P^{r}\left(M_{g}\right) \times L E_{i} \longrightarrow L E_{i+r}
$$

described earlier, and suppo $i \geq 2 g$; then

$$
U_{i+k} \mapsto b^{k} \ll \frac{2^{k}}{k !}\left(\sum_{1}^{g} f_{2 r-1} f_{2 r}\right)^{k} U_{i},
$$

where $U_{j}$ is the generating cliss above for $H^{2(j-g)}\left(L E_{j} ; \mathbf{Z}\right)$ when $n=1$. 


\section{Stabilization}

For $d \geq g$, any $n \geq 1$ and any neighborhood $U_{\epsilon}(*)$, there are $n+1$ divisors $D_{i} \subset S P^{d}\left(U_{\epsilon}(*)\right), 0 \leq i \leq n$ with $\mu\left(D_{0}\right)=\mu\left(D_{1}\right)=\cdots=$ $\mu\left(D_{n}\right)$ and $\bigcap D_{i}=\emptyset$. By deforming $M_{g}-*$ to $M_{g}-U_{\epsilon}$ and then adding in the corresponding divisors, one obtains a stablization map $\tau$ which one can iterate

$$
\operatorname{Hol}_{k}^{*}\left(M_{g}, \mathbf{P}^{n}\right) \stackrel{\tau}{\longrightarrow} \operatorname{Hol}_{k+d}^{*}\left(M_{g}, \mathbf{P}^{n}\right) \longrightarrow \cdots \longrightarrow \operatorname{Hol}_{k+r d}^{*}\left(M_{g}, \mathbf{P}^{n}\right)
$$

obtaining in the limit a space $\lim _{r \mapsto \infty} \operatorname{Hol}_{k+r d}^{*}\left(M_{g}, \mathbf{P}^{n}\right)$ which is homotopy equivalent to any component in the mapping space $\operatorname{Map}_{*}^{*}\left(M_{g}, \mathbf{P}^{n}\right)$. Note that this last statement is a consequence of Segal's stabilization theorem, [21].

The inclusion $\tau$ descends to a map of spectral sequences and by an argument similar to one in [5], we see the corresponding map on the $E_{1}$-term is given by

$$
\begin{aligned}
& \cup\left(b_{0} b_{1} \cdots b_{n}\right) \otimes i d: \\
& \quad H^{*}\left(L E_{i} ; \mathbf{Z}\right) \otimes H^{*}\left(S P^{j}\left(\Sigma M_{g}\right), S P^{j-1}\left(\Sigma M_{g}\right) ; \mathbf{Z}\right) \\
& \longrightarrow H^{*}\left(L E_{i+1} ; \mathbf{Z}\right) \otimes H^{*}\left(S P^{j}\left(\Sigma M_{g}\right), S P^{j-1}\left(\Sigma M_{g}\right) ; \mathbf{Z}\right)
\end{aligned}
$$

This relation between inclusion at the level of mapping spaces and cupping with $b_{0} \cdots b_{n}$ in the Poincare duals is quite important and follows basically by checking normal bundles (cf [12]). In any case the content of the preceeding remarks can be summarized in

Lemma 8.3. In the stable range the cohomology of $\mathrm{Hol}_{k}^{*}\left(M_{g}, \mathbf{P}^{n}\right)$ is isomorphic to the cohomology of $\operatorname{Map}_{k}^{*}\left(M_{g}, \mathbf{P}^{n}\right)$ via the natural inclusion.

This is again direct from the stabilization via cupping with $b_{0} \cdots b_{n}$ and Segal's stability theorem.

The differentials which we know appear come from comparison with the $D i v$-space spectral sequence. These are the differential $d_{1}$ already described and the further differentials which only $\operatorname{hold} \bmod (p)$ for odd $p$

$$
\begin{aligned}
d\left(\left|\gamma_{p^{i}}\right|^{*}\right) & =\frac{1}{p^{i}}\left(\sum_{1}^{g} f_{2 j-1} f_{2 j}\right)^{p^{i}} \\
d\left(\left.\left|\gamma_{p^{i}}^{p-1}\right| \gamma_{p^{i}}\right|^{*}\right) & =\left[\frac{1}{p^{i}}\left(\sum_{1}^{g} f_{2 j-1} f_{2 j}\right)^{(p-1) p^{i}}\right]\left|\gamma_{p^{i}}\right|^{*}
\end{aligned}
$$


The last differential in (8.4) is called the Kudo differential, [14]. There are no differentials for $p=2$ (see next remark). Similarly, for $n>2$ all the differentials are zero for all $p$ and hence, even over the integers.

Remark 8.5. There is a sequence of Serre fibrations

$$
\Omega^{2}\left(\mathbf{P}^{n}\right) \longrightarrow \operatorname{Map}^{*}\left(M_{g}, \mathbf{P}^{n}\right) \longrightarrow\left(\Omega\left(\mathbf{P}^{n}\right)\right)^{2 g} \stackrel{f^{\dagger}}{\longrightarrow} \Omega\left(\mathbf{P}^{n}\right)
$$

induced from the cofibration sequence associated to $M_{g}$;

$$
S^{1} \stackrel{f}{\rightarrow} \bigvee^{2 g} S^{1} \hookrightarrow M_{g} \longrightarrow S^{2}
$$

which gives $\operatorname{Map}^{*}\left(M_{g}, \mathbf{P}^{n}\right)$ as the total space of a principal $\Omega^{2}\left(\mathbf{P}^{n}\right)$ fibration with classifying map $f^{\dagger}$. Then the discussion above shows by dualizing that in the Serre spectral sequence of the fibration

$$
\Omega^{2}\left(\mathbf{P}^{n}\right) \longrightarrow \operatorname{Map}^{*}\left(M_{g}, \mathbf{P}^{n}\right) \longrightarrow\left(\Omega\left(\mathbf{P}^{n}\right)\right)^{2 g}
$$

we have (for $n=1$ ) the differentials $d^{1}\left(|b|^{*}\right)=2 \sum_{1}^{g} f_{2 j-1} f_{2 j}$, the transgressive $\bmod (p)$ differentials from $\left|b^{p^{i}}\right|^{*}$ to the divided power of $\left(\sum_{1}^{g} f_{2 j-1} f_{2 j}\right)$, and the Kudo differentials for $p$ odd. Of course, for $p=2$ the differentials in the Serre spectral sequence are totally trangressive from the fiber to the base. Hence, there are no differentials and $E_{2}=E_{\infty}$. Similarly, for $n>2$ there are no differentials for any $p$ and $E_{2}=E_{\infty}$ in this spectral sequence even with integer coefficients though the only way we know to prove this is via the results of [13].

\section{The genus one case}

When $g=1$, then $\mu: M_{1} \rightarrow J\left(M_{1}\right)$ is a holomorphic homeomorphism identifying the Jacobi variety with $T$ itself. Also, the stable range starts immediately in this case and hence, for all $n \geq 1$ the Abel-Jacobi map $\mu$ is a fibration

$$
\mathbf{P}^{n-1} \longrightarrow S P^{n}(T) \stackrel{\mu}{\longrightarrow} T \text {. }
$$

In the spectral sequence for $Q E_{k}(n)$ with $n \geq 2\left(\right.$ dual to $H o l_{k}^{*}\left(T, \mathbf{P}^{n}\right)$ ) there are no differentials except for those at the tail end (6.15) and (6.16), but these are the only differentials and $E^{1}=E^{\infty}$ away from this region, while $E^{2}=E^{\infty}$ for the entire spectral sequence. In the case $n=1$ the only differential is again $d_{1}$ which now also has a stable component given in (5.9), and again $E^{2}=E^{\infty}$. 
It also follows that duality gives the isomorphism

$$
\tilde{H}^{2 k(n+1)-2 n-*}\left(\operatorname{Hol}_{k}^{*}\left(T, \mathbf{P}^{n}\right) ; \mathbf{F}\right) \cong H_{*}\left(Q E_{E_{n+1}^{k, \ldots, k}} ; \mathbf{F}\right)
$$

for all $k \geq 2$, with $H_{o l}^{*}\left(T, \mathbf{P}^{n}\right)$ being empty for all $n$.

We now determine these homology groups explicitly for $n=1$. First, in the case where $\mathbf{F}$ has characteristic 2 there are no stable differentials and $E^{1}=E^{\infty}$ in this range. Second, in case $\mathbf{F}=\mathbf{Q}$ we have

$$
\begin{aligned}
E^{1}\left(\operatorname{Hol}_{k}^{*}\right)= & \coprod_{i=2}^{k} \Sigma^{4(i-1)} H_{*}(T ; \mathbf{Q}) \\
& \otimes\left(\mathbf{Q}\left[\left|e_{1}\right|,\left|e_{2}\right|\right]_{k-i} \oplus|[T]| \otimes \mathbf{Q}\left[\left|e_{1}\right|,\left|e_{2}\right|\right]_{k-i-1}\right) \\
& \oplus \tilde{H}_{*}(T ; \mathbf{Q}) \otimes\left(\mathbf{Q}\left[\left|e_{1}\right|,\left|e_{2}\right|\right]_{k-1} \oplus|[T]| \otimes \mathbf{Q}\left[\left|e_{1}\right|,\left|e_{2}\right|\right]_{k-2}\right) \\
& \oplus \mathbf{Q}\left[\left|e_{1}\right|,\left|e_{2}\right|\right]_{k}+|T| \mathbf{Q}\left[\left|e_{1}\right|,\left|e_{2}\right|\right]_{k-1},
\end{aligned}
$$

where $\mathbf{Q}\left[\left|e_{1}\right|,\left|e_{2}\right|\right]_{r}$ is the $(r+1)$-dimensional subspace spanned by the monomials of degree $r$; that is: $\left|e_{1}\right|^{r},\left|e_{1}\right|^{r-1}\left|e_{2}\right|, \ldots,\left|e_{2}\right|^{r}$. The only differential is $d_{1}$, generated by

$$
d_{1}\left(\Sigma^{4(k-j-1)} e_{1} e_{2}|T|\right)=\Sigma^{4(k-j)} 1,
$$

and the unstable differentials of (6.15) and (6.16). Thus one has directly (compare (6.19) for the last term) that

$$
\begin{aligned}
E^{2}= & \coprod_{i=2}^{k} \Sigma^{4(i-1)} \tilde{H}_{*}(T ; \mathbf{Q}) \otimes \mathbf{Q}\left[\left|e_{1}\right|,\left|e_{2}\right|\right]_{k-i} \\
& \oplus \Sigma^{4(i-1)}|T|\left(1, e_{1}, e_{2}\right) \mathbf{Q}\left[\left|e_{1}\right|,\left|e_{2}\right|\right]_{k-i-1} \\
& \oplus \mathbf{Q}\left\{e_{2}\left|e_{1}\right|^{k-1}, e_{2}\left|e_{1}\right|^{k-2}\left|e_{2}\right|, \ldots, e_{2}\left|e_{1}\right|\left|e_{2}\right|^{k-2}\right\} \\
& \oplus|T|\left(1, e_{1}, e_{2}\right) \mathbf{Q}\left[\left|e_{1}\right|,\left|e_{2}\right|\right]_{k-2} \\
= & E^{\infty}
\end{aligned}
$$

In the remaining case where $\mathbf{F}=\mathbf{F}_{p^{n}}$ has characteristic $\boldsymbol{p}$ an odd prime, as explained in (6.6), (6.7) we can write the $E_{1}$-term as

$$
\coprod_{i+j=k} \Sigma^{4(i-1)} H^{*}(T ; \mathbf{F}) \otimes \sum_{l=0}^{j} \Sigma^{4 l} \mathcal{D}_{j}^{*}(p) \otimes \mathbf{F}\left[\mid b_{1}, b_{2}\right]_{j-l},
$$

where $\mathcal{D}_{j}^{*}=0$ if $j \neq p k$ or $p k+1$ and with the usual special considerations at the tail end of the filtration. Incidently, the case $j=p k+1$ 
implies that $\Sigma^{4 j} \mathcal{D}_{j}^{*}=|M| \Sigma^{4(j-1)} \mathcal{D}_{j-1}^{*}$. The differential is as before, and we have that in the stable range

$$
\begin{aligned}
E^{2} & =\coprod_{i=0}^{k} \Sigma^{4(i+j-1)} \tilde{H}_{*}(T ; \mathbf{F}) \otimes \mathcal{D}_{j}^{*} \otimes \mathbf{F}\left[b_{1}, b_{2}\right]_{k-i-j} \\
& =E^{\infty} .
\end{aligned}
$$

In each case the homology injects into $H_{*}\left(\operatorname{Map}_{k}^{*}\left(T, \mathbf{P}^{1}\right) ; \mathbf{F}\right)$. Thus the geometric interpretation of each of the homology and cohomology classes in $H_{*}\left(\operatorname{Hol}_{k}^{*}\left(T, \mathbf{P}^{1}\right) ; \mathbf{F}\right)$ can be regarded as the same as that for the corresponding class in the mapping space.

For example with rational coefficients we have the following corollary to 9.3 and 9.1 .

Lemma 9.4. Let $I=\left(\left|e_{1}\right|,\left|e_{2}\right|\right)$ be the augmentation ideal in $\mathbf{Q}\left[\left|e_{1}\right|,\left|e_{2}\right|\right]$. Then

$$
\begin{aligned}
H_{*}\left(\operatorname{Hol}_{k}^{*}\left(T, \mathbf{P}^{1}\right) ; \mathbf{Q}\right) \cong & \mathbf{Q}\left(1, e_{1}, e_{2}, h_{2,1}, h_{2,2}, v_{3}\right) \otimes \mathbf{Q}\left[\left|e_{1}\right|,\left|e_{2}\right|\right] / I^{k-1} \\
& \oplus \mathbf{Q}\left\{e_{2}\left|e_{1}\right|^{k-1}, \ldots, e_{2}\left|e_{1}\right|\left|e_{2}\right|^{k-2}\right\}
\end{aligned}
$$

In particular the Poincaré series for $H_{*}\left(\operatorname{Hol}_{k}^{*}\left(T, \mathbf{P}^{1}\right) ; \mathbf{Q}\right)$ is

$$
\left(1+2 x+2 x^{2}+x^{3}\right)\left(1+2 x^{2}+3 x^{4}+\cdots+(k-1) x^{2(k-2)}\right)+(k-2) x^{2(k-1)-1} .
$$

Note how this compares with the corresponding Poincare series for the mapping space $H_{*}\left(\operatorname{Map}^{*}\left(T, \mathbf{P}^{1}\right) ; \mathbf{Q}\right)$ :

$$
\frac{\left(1+2 x+2 x^{2}+x^{3}\right)}{\left(1-x^{2}\right)^{2}} \text {. }
$$

The case $n \geq 2$. Any component in the mapping space is given as the total space of the Serre fibration

$$
\Omega^{2} S^{2 n+1} \rightarrow E \rightarrow\left(S^{1} \times \Omega S^{2 n+1}\right)^{2}
$$

with

$$
H_{*}\left(\operatorname{Map}^{*}\left(T, \mathbf{P}^{n}\right) ; \mathbf{F}\right)=H_{*}\left(\Omega^{2} S^{2 n+1} ; \mathbf{F}\right) \otimes H_{*}(T ; \mathbf{F}) \otimes \mathbf{F}\left[g_{2 n}, g_{2 n}^{\prime}\right]
$$


Consequently, with $\mathbf{Q}$ as coefficients the Poincare series for the mapping space has the form

$$
\frac{(1+x)^{2}\left(1+x^{2 n-1}\right)}{\left(1-x^{2 n}\right)^{2}}
$$

while for $\operatorname{Hol}_{k}^{*}\left(T, \mathbf{P}^{n}\right)$ we have

$$
(1+x)^{2}\left(1+x^{2 n-1}\right)\left(1+2 x^{2 n}+3 x^{4 n}+\cdots+(k-1) x^{(k-2) 2 n}\right)+(k-2) x^{2(k-1) n-1} .
$$

Remark 9.5. Notice that in this case all the calculations were independent of the particular elliptic curve $T$ that we were studying, even though different $T$ are not holomorphically isomorphic. In fact this is not unexpected since we have

Theorem 9.6. Let $T$ be any non-singular elliptic curve. Then the homeomorphism type of $\operatorname{Hol}_{k}^{*}\left(T, \mathbf{P}^{n}\right)$ is independent of $T$.

Proof. Let $T$ and $T^{\prime}$ be two given tori with corresponding lattices $\Lambda$ and $\Lambda^{\prime} \subset \mathbf{C}$. Then there is a map $\Lambda \longrightarrow \Lambda^{\prime}$ which induces a homeomorphism

$$
T=\mathbf{C} / \Lambda \stackrel{f}{\rightarrow} T^{\prime}=\mathbf{C} / \Lambda^{\prime},
$$

and such that the following commutes for all $n$;

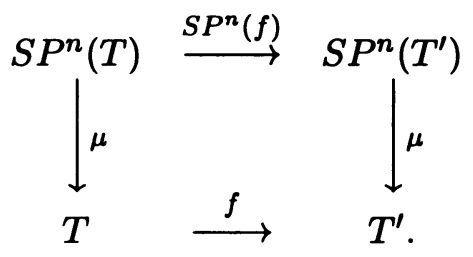

It follows therefore that $E_{i, i, \ldots i} \cong E_{i, i, \ldots, i}^{\prime}$ where $E_{i, i, \ldots i}$ (resp. $E_{i, i, \ldots, i}^{\prime}$ ) is the pushout as in $\S 3$

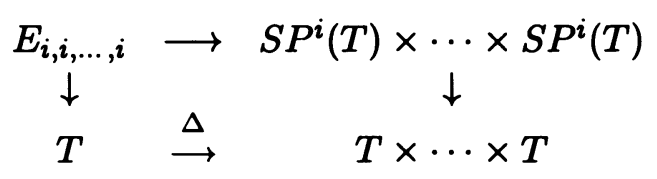

(respectively, $T$ is replaced by $T^{\prime}$ ). It is now clear that

$$
\operatorname{Hol}_{n}^{*}\left(T, \mathbf{P}^{n}\right) \cong \tilde{E}_{i, \ldots i}-\operatorname{Image}(\nu) \cong \tilde{E}_{i, \ldots i}^{\prime}-\operatorname{Image}(\nu) \cong \operatorname{Hol}_{n}^{*}\left(T, \mathbf{P}^{n}\right),
$$

and the proof is complete. q.e.d. 


\section{The decomposition for hyperelliptic curves}

The surface $M_{g}$ is hyperelliptic if and only if there is a degree-two (branched) holomorphic map $f: M_{g} \rightarrow \mathbf{P}^{1}$. This map $f$ fits in the following diagram

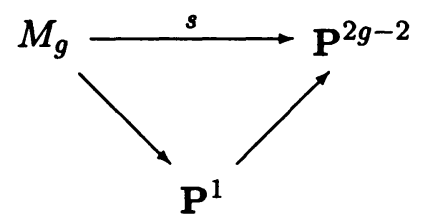

where $s$ is the canonical map, [8, p. 247].

From the point of view of the Abel-Jacobi maps, $M_{g}$ is hyperelliptic if and only if we have a cofibering of the form

$$
\mathbf{P}^{1} \hookrightarrow S P^{2}\left(M_{g}\right) \longrightarrow S P^{2}\left(M_{g}\right) / \mathbf{P}^{1}=W_{2} \subset J\left(M_{g}\right) .
$$

The holomorphic embedding $\mathbf{P}^{1} \hookrightarrow S P^{2}\left(M_{g}\right)$ is now constructed by associating to $z \in \mathbf{P}^{1}$ the pair $f^{-1}(z) \in S P^{2}\left(M_{g}\right)$ where the points are counted with multiplicity, i.e., the ramification points are counted twice. The image $\tau \in J\left(M_{g}\right)$ of $\mathbf{P}^{1}$ under $\mu_{2}$ is called the hyperelliptic point of $M_{g}$.

Lemma 10.2. Suppose that $M_{g}$ is hyperelliptic and $\tau \in J\left(M_{g}\right)$ is the hyperelliptic point. Let $k \geq 1$ and $t \leq\left[\frac{k}{2}\right]$. Then:

(a) for $k \leq g$, the space $W_{k}^{t}$ is $\mu_{k-2 t}\left(S P^{k-2 t}\left(M_{g}\right)\right)+t \tau$,

(b) for $2 g-1>k>g, t>k-g$, we also have

$$
W_{k}^{t}=\mu_{k-2 t}\left(S P^{k-2 t}\left(M_{g}\right)\right)+t \tau .
$$

Proof. Note that $S P^{r}\left(\mathbf{P}^{1}\right)=\mathbf{P}^{r}$ so the pairing

$$
S P^{2 l}\left(M_{g}\right) \times S P^{k-2 l}\left(M_{g}\right) \longrightarrow S P^{k}\left(M_{g}\right)
$$

induces an action

$$
\nu: \mathbf{P}^{l} \times S P^{k-2 l}\left(M_{g}\right) \stackrel{=}{\longrightarrow} S P^{l}\left(\mathbf{P}^{1}\right) \times S P^{k-2 l}\left(M_{g}\right) \longrightarrow S P^{k}\left(M_{g}\right),
$$

and the image of

$$
\nu\left(\left\langle p_{1}, \ldots, p_{l}\right\rangle,\left\langle m_{1}, \ldots, m_{k-2 l}\right\rangle\right)
$$


under the Abel-Jacobi map is $l \tau+\mu_{k-2 l}\left(\left\langle m_{1}, \ldots, m_{k-2 l}\right\rangle\right.$. From this follows directly the fact that $W_{k}^{t}$ is at least as large as asserted in (a). The inclusion argument for (b) is a similar dimension check when we recall that generically $\mu_{g+s}^{-1}(j)=\mathbf{P}^{s}$, so we are checking for points with inverse image $\mathbf{P}^{t}$ where $t>s$ and $\nu\left(\mathbf{P}^{t} \times S P^{g+s-2 t}\left(M_{g}\right)\right) \subset \mu_{g+s}^{-1}\left(W_{g+s}^{t}\right)$.

The converse is given on page 13 of [1] for $k \leq g$ where it is pointed out that as a consequence of the geometric version of the RiemannRoch Theorem every $\mathbf{P}^{t}$ inverse image under the Abel-Jacobi map for $S P^{k}\left(M_{g}\right)$ has the form $\mu^{-1}(t \tau)+m_{1}+\cdots+m_{k-2 t}$ where no two of the $m_{i}$ 's are conjugate in $M_{g}$ under the hyperelliptic involution. To obtain the remaining part of the converse one uses the residual series isomorphism

$$
W_{k}^{t} \cong W_{2 g-2-k}^{g-k+t-1}, \quad D \leftrightarrow K-D,
$$

which holds for all curves $M_{g}, k \geq g$. q.e.d.

For later use we also specify an appropriate base point for the construction. Let $* \in M_{g}$ be one of the ramification points of $f: M_{g} \rightarrow \mathbf{P}^{1}$. Then $2 \mu(*)=\tau$, and we choose $*$ as the base point in $M_{g}$. Also the base point of $\mathbf{P}^{1}$ is $\langle *, *\rangle$, and the inclusion $f: \mathbf{P}^{1} \rightarrow S P^{2}\left(M_{g}\right)$ is based. With respect to this choice, the composite inclusion

$$
S P^{k}\left(M_{g}\right) \hookrightarrow S P^{k+1}\left(M_{g}\right) \hookrightarrow S P^{k+2}\left(M_{g}\right)
$$

commutes with the $\mathbf{P}^{1}$-action. Moreover, in the actual construction of the Abel-Jacobi map $\mu$ given at the beginning of $\S 1$ we can take our base point in $M_{g}$ to be $*$, so that $\mu(*)=0 \in J\left(M_{g}\right)$, and the hyperelliptic point $\tau=0$ as well. This has the advantage that the inclusion $W_{k}^{t}+\tau \subset W_{k+2}^{t+1}$ becomes simply $W_{k}^{t} \subset W_{k+1}^{t+1}$.

\section{Some preliminary $\cdot$ constructions}

Let $V$ be a space with a commutative, associative multiplication. For example

$$
V=S P^{\infty}(X, *), V=\coprod_{1}^{\infty} S P^{n}(X), V=\coprod_{1}^{\infty} S P^{i}(X) \times S P^{i}(X),
$$

etc. If $f: X \rightarrow V$ is any continuous map, then $f$ defines an action

$$
\mu_{f}: \coprod S P^{k}(X) \times V \rightarrow V
$$


by $\mu_{f}\left(\left\langle x_{1}, \ldots, x_{k}\right\rangle, v\right)=f\left(x_{1}\right) f\left(x_{2}\right) \cdots f\left(x_{k}\right) v$.

Suppose now that $V, f_{1}: \mathbf{P}^{1} \rightarrow V$ and $V^{\prime}, f_{2}: \mathbf{P}^{1} \rightarrow V^{\prime}$ are given with $V, V^{\prime}$ as above. Then we can form the bicomplex

$$
B\left(f_{1}, f_{2}\right):=V \times_{f_{1}}\left(\coprod_{k} S P^{k}\left(I \times \mathbf{P}^{1}\right)\right) \times_{f_{2}} V^{\prime},
$$

defined by the equivalence relation built from the basic relations

$$
\begin{aligned}
\left(v,\left\langle\left(t_{1}, p_{1}\right), \ldots,\left(t_{k}, p_{k}\right)\right\rangle, v^{\prime}\right) \\
\sim \begin{cases}\left(v_{1} \cdot f_{1}\left(p_{i}\right),\left\langle\left(t_{1}, p_{1}\right), \ldots, \widehat{\left(t_{i}, p_{i}\right)}, \ldots,\left(t_{k}, p_{k}\right)\right\rangle, v^{\prime}\right) & \text { if } t_{i}=0 \\
\left(v_{1},\left\langle\left(t_{1}, p_{1}\right), \ldots, \widehat{\left(t_{i}, p_{i}\right)}, \ldots,\left(t_{k}, p_{k}\right)\right\rangle, f_{2}\left(p_{i}\right) \cdot v^{\prime}\right) & \text { if } t_{i}=1\end{cases}
\end{aligned}
$$

Remark 11.2. This is just the 2-sided bar construction which is associated to the homological functor $\operatorname{Tor}_{A}\left(M_{A},{ }_{A} N\right)$. But here, left and right modules are equivalent since $A$ is commutative.

Remark 11.3. This is an unbased construction. If $f_{1}$ and $f_{2}$ are based maps with $f_{i}(*)=i d, i=1,2$, then there is an associated reduced construction, $\tilde{B}\left(f_{1}, f_{2}\right)$. Also, the fact that we are using $\mathbf{P}^{1}$ plays no real role in the definition. It is just there because that is all we use in the applications of this construction to our study of $\mathrm{Hol}_{k}^{*}\left(M_{g}, \mathrm{P}^{n}\right)$ for $M_{g}$ hyperelliptic.

Example 11.4. Suppose that $V=\coprod S P^{k}(X)$ and

$$
f_{1}: \mathbf{P}^{1} \rightarrow S P^{i}(X)
$$

Then write

$$
V_{j}=\coprod_{k=0}^{\infty} S P^{j+i k}(X), \quad 0 \leq j<i
$$

so we have separate actions on each $V_{j}$.

Example 11.5. Suppose $f_{2}: \mathbf{P}^{1} \rightarrow *$ where $*$ has the obvious commutative and associative action. Then

$$
B\left(f_{1}, f_{2}\right)=V \times_{f_{1}} \coprod S P^{n}\left(I \times \mathbf{P}^{1}\right) \times_{f_{2}} * \simeq V \times_{f_{1}} \coprod S P^{n}\left(c \mathbf{P}^{1}\right)
$$

is a space that we have seen many times before. 
Example 11.6. $\Delta_{n+1}: \mathbf{P}^{1} \longrightarrow\left(\mathbf{P}^{1}\right)^{n+1}$ induces an action on $\left(\amalg S P^{k}\left(\mathbf{P}^{1}\right)\right)^{n+1}$ which restricts, in a manner similar to that in example (1) to an action on

$$
\mathcal{E}_{n}\left(i_{1}, \ldots, i_{n+1}\right)=\coprod_{k=0}^{\infty} S P^{i_{1}+k}\left(\mathbf{P}^{1}\right) \times \cdots \times S P^{i_{n+1}+k}\left(\mathbf{P}^{1}\right)
$$

for each $(n+1)$-tuple $\left(i_{1}, \ldots, i_{n+1}\right)$ of non-negative integers with at least one of the $i_{j}=0$.

12. Models for the spaces $E_{i, \ldots, i}, L E_{i, \ldots, i}$ and $W_{k}^{t}$ when $M_{g}$ is hyperelliptic

We specialize to

$$
\begin{gathered}
B H(f, n) \\
=\left(\coprod_{m} S P^{m}\left(M_{g}\right)\right) \times{ }_{f}\left(\coprod_{m} S P^{m}\left(I \times \mathbf{P}^{1}\right)\right) \\
\times_{f_{2}} \mathcal{E}_{n}(0,0, \ldots, 0),
\end{gathered}
$$

where $f: \mathbf{P}^{1} \hookrightarrow S P^{2}\left(M_{g}\right)$ embeds $\mathbf{P}^{1}$ as the inverse image under the Abel-Jacobi map of the hyperelliptic point, 0 , for some hyperelliptic structure on $M_{g}$, and $f_{2}$ is the structure map $\Delta_{n+1}$ of (11.6).

This construction specializes, as in (11.5), to give two disjoint subspaces,

$$
\begin{aligned}
& B H_{0}(f, n)=V_{0} \times_{f}\left(\coprod_{m} S P^{m}\left(I \times \mathbf{P}^{1}\right)\right) \times_{f_{2}} \mathcal{E}_{n}(0, \ldots, 0), \\
& B H_{1}(f, n)=V_{1} \times_{f}\left(\coprod_{m} S P^{m}\left(I \times \mathbf{P}^{1}\right)\right) \times_{f_{2}} \mathcal{E}_{n}(0, \ldots, 0),
\end{aligned}
$$

where $V_{0}=\amalg S P^{2 k}\left(M_{g}\right)$ and $V_{1}=\amalg S P^{2 k+1}\left(M_{g}\right)$. Each $B H_{\epsilon}(f, n)$ breaks up into further components as follows. We define a grading by letting

$$
\begin{array}{r}
\left(\left\langle m_{1}, \ldots, m_{l}\right\rangle,\left\langle\left(t_{1}, p_{1}\right), \ldots,\left(t_{r}, p_{r}\right)\right\rangle,\left(w_{s, 1}, \ldots, w_{s, n+1}\right)\right) \\
\in S P^{l}\left(M_{g}\right) \times S P^{r}\left(I \times \mathbf{P}^{1}\right) \times \prod_{1}^{n+1} S P^{s}\left(\mathbf{P}^{1}\right)
\end{array}
$$


have grading degree $l+2 r+2 s$. This grading is obviously preserved by the identifications and hence induces the desired decomposition of the quotient $B H_{\epsilon}(f)$. We write $\mathcal{G}_{s}(f, n)$ for the component of points of grading degree $s$.

This is the general case, however, there is a special case $B H_{\epsilon}(f,-1)$ which is also needed where $V_{2} \sim *$ consists of a single point. In this case the grading degree is not preserved by the identifications, and the grading only gives a filtration of $B H_{\epsilon}(f,-1)$. We write $\mathcal{F}_{k}\left(B H_{\epsilon}(f,-1)\right)$ for the filtering subspace consisting of all points of grading degree $\leq k$. Moreover, in this case the identification has the form $(v,(t, x), *) \sim$ $(v f(x), *)$ when $t=0$ and $(v,(t, x), *) \sim(v, *)$ when $t=1$.

Lemma 12.5. We have the following homotopy equivalences in the case where $M_{g}$ is hyperelliptic:

(a) $\mathcal{G}_{k}\left(B H_{\epsilon}(f, n)\right) \simeq E_{k, \ldots, k}$ for $k \equiv \epsilon \bmod (2)$ provided $k \leq g$.

(b) $\mathcal{F}_{k}\left(B H_{\epsilon}(f,-1)\right) \simeq W_{k}, k \equiv \epsilon \bmod (2)$ and $k<g$.

(These constructions are modeled on the analogous constructions in [20] and the proofs are the same.)

Remark 12.6. The point of these constructions is to handle the algebraic complexities of systematically collapsing out $\mathbf{P}^{n}$ 's. As one sees from the lemma, the effect is to introduce $S P^{n}\left(\Sigma \mathbf{P}^{1}\right)$ 's which provide a measure of the geometry of the collapsed spaces as the collapsing gets more and more complex.

\section{Some spectral sequences for the $B H_{\epsilon}(f, n)$}

We can bifilter $\mathcal{G}_{k}\left(B H_{\epsilon}(f, n)\right)$ and $\mathcal{F}_{k}\left(B H_{\epsilon}(f,-1)\right)$ by the number of $S P^{t}\left(I \times \mathbf{P}^{1}\right)$ terms appearing, obtaining spectral sequences converging to the various spaces above as well as certain useful quotients. The $E^{1}$-terms are as follows:

$$
\coprod_{k+2 l+2 t=v} H_{*}\left(S P^{k}\left(M_{g}\right)\right)
$$

$$
\otimes H_{*}\left(S P^{l}\left(\Sigma \mathbf{P}_{+}^{1}\right), S P^{l-1}\left(\Sigma \mathbf{P}_{+}^{1}\right)\right) \otimes\left(H_{*}\left(\mathbf{P}^{t}\right)\right)^{n+1}
$$

converging to $H_{*}\left(E_{v, \ldots, v}\right)$ with field coefficients and $v<g$,

$$
\coprod_{k+2 l \leq v} H_{*}\left(S P^{k}\left(M_{g}\right)\right) \otimes H_{*}\left(S P^{l}\left(\Sigma \mathbf{P}_{+}^{1}\right), S P^{l-1}\left(\Sigma \mathbf{P}_{+}^{1}\right)\right)
$$


with $k \equiv v \bmod (2)$ converging to $H_{*}\left(W_{v}\right)$ for $v<g$, and

$$
\coprod_{k+2 l=v} H_{*}\left(S P^{k}\left(M_{g}\right)\right) \otimes H_{*}\left(S P^{l}\left(\Sigma \mathbf{P}_{+}^{1}\right), S P^{l-1}\left(\Sigma \mathbf{P}_{+}^{1}\right)\right)
$$

converging to $H_{*}\left(W_{v} / W_{v}^{1}\right)$. (The notation $\Sigma \mathbf{P}_{+}^{1}$ means the reduced suspension on the union of $\mathbf{P}^{1}$ with a disjoint base point denoted + , it has the homotopy type of the wedge $\Sigma\left(\mathbf{P}^{1}\right) \vee S^{1}$.)

If we relativize we obtain a spectral sequence with $E^{1}$-term

$$
\begin{gathered}
\coprod_{k+2(l+t)=v} H_{*}\left(S P^{k}\left(M_{g}\right), S P^{k-1}\left(M_{g}\right)\right) \\
\otimes H_{*}\left(S P^{l}\left(\Sigma \mathbf{P}_{+}^{1}\right), S P^{l-1}\left(\mathbf{P}_{+}^{1}\right)\right) \otimes \tilde{H}_{*}\left(S^{2(n+1) t}\right) \\
\Longrightarrow \tilde{H}_{*}\left(L E_{v, \ldots, v}\right) .
\end{gathered}
$$

All these spectral sequences are algebraic in the sense of [12], and can be modeled by simply replacing the terms $H_{*}\left(S P^{l}\left(\Sigma \mathbf{P}^{1}\right)\right)$ by the cobar construction on

$$
H_{*}\left(\coprod S P^{l}\left(\mathbf{P}_{+}^{1}\right)\right)=\mathbf{Z}[t] \otimes \Gamma(b) .
$$

In particular, the relative spectral sequence splits as a direct sum of spectral sequences each of the form

$$
\begin{gathered}
{\left[\coprod_{k+2 l=v-2 t} H_{*}\left(S P^{k}\left(M_{g}\right), S P^{k-1}\left(M_{g}\right)\right) \otimes H_{*}\left(S P^{l}\left(\Sigma P_{+}^{1}\right), S P^{l-1}\left(\Sigma P_{+}^{1}\right)\right)\right]} \\
\otimes \tilde{H}_{*}\left(S^{2 t(n+1)}\right) \\
\Longrightarrow \Sigma^{2 t(n+1)}\left(H_{*}\left(W_{v-2 t} /\left(W_{v-2 t}^{1} \cup W_{v-2 t-1}\right)\right)\right)
\end{gathered}
$$

for any $n>0$.

In the case where the coefficients are the rationals, these spectral sequences become quite simple. First $H_{*}\left(S P^{\infty}\left(\Sigma \mathbf{P}_{+}^{1}\right) ; \mathbf{Q}\right)=\Lambda\left(h_{1}, h_{3}\right)$, and

$$
H_{*}\left(S P^{i}\left(\Sigma \mathbf{P}^{1}\right), S P^{i-1}\left(\Sigma \mathbf{P}^{1}\right) ; \mathbf{Q}\right)=0
$$

for $i \geq 3$. Second, when we write

$$
H_{*}\left(S P^{k}\left(M_{g}\right), S P^{k-1}\left(M_{g}\right) ; \mathbf{Z}\right)=\bigoplus_{s=0}^{k} \Lambda_{s}\left(e_{1}, \ldots, e_{2 g}\right) \otimes\left[M_{g}\right]^{k-s},
$$


for $k<g$, we see that the action map

$$
\begin{aligned}
*\left[\mathbf{P}^{1}\right]: & H_{*}\left(S P^{k}\left(M_{g}\right), S P^{k-1}\left(M_{g}\right) ; \mathbf{Z}\right) \\
& \rightarrow H_{*+2}\left(S P^{k+2}\left(M_{g}\right), S P^{k-1}\left(M_{g}\right) ; \mathbf{Z}\right)
\end{aligned}
$$

is just multiplication by $-\sum_{j=1}^{g} e_{2 j-1} e_{2 j}$ since

$$
\left[\mathbf{P}^{1}\right]=\left[M_{g}\right]-\sum_{1}^{g} e_{2 j-1} e_{2 j}
$$

in $H_{*}\left(S P^{2}\left(M_{g}\right) ; \mathbf{Z}\right)$. Third, from [2], this map is injective in homology for $k \leq g-2$. Thus we have

\section{Lemma 13.6.}

$$
\begin{aligned}
H_{*}\left(L E_{v, \ldots, v} ; \mathbf{Q}\right) & \\
& =\sum_{t=0}^{[v / 2]} \frac{\Sigma^{2 t(n+1)} H_{*}\left(S P^{v-2 t}\left(M_{g}\right), S P^{v-2 t-1}\left(M_{g}\right) ; \mathbf{Q}\right)}{i m\left(*\left(\sum_{j=1}^{g} e_{2 j-1} e_{2 j}\right)\right)}
\end{aligned}
$$

for $v \leq g$, which has Poincaré series

$$
\sum_{t=0}^{[v / 2]} x^{2 t(n+1)} S_{v-2 t},
$$

where $S_{r}=\sum_{l \leq r}\left[\left(\begin{array}{c}2 g \\ l\end{array}\right)-\left(\begin{array}{c}2 g \\ l-1\end{array}\right)\right] x^{2 r-l}$.

Similarly, we can analyze $H_{*}\left(W_{j} ; \mathbf{Q}\right)$.

\section{Lemma 13.7.}

(a) The inclusion $W_{j} \subset J\left(M_{g}\right)$ induces an injection in rational homology $H_{*}\left(W_{j} ; \mathbf{Q}\right) \hookrightarrow H_{*}\left(J\left(M_{g}\right) ; \mathbf{Q}\right)=\Lambda\left(e_{1}, \ldots, e_{2 g}\right)$ with image the subvector space spanned by the subspaces

$$
\left\{\Lambda_{s}\left(e_{1}, \ldots, e_{2 g}\right)\left[M_{g}\right]^{t} \mid s+t \leq j\right\},
$$

where $\left[M_{g}\right]=\sum_{1}^{g} e_{2 i-1} e_{2 i}$ is the image of the fundamental class of $M_{g}$ under the Abel-Jacobi map $\mu_{*}$,

(b) $H_{*}\left(W_{j-1} ; \mathbf{Q}\right)$ injects into $H_{*}\left(W_{j} ; \mathbf{Q}\right)$ under the inclusion, so the relative groups are given as

$$
H_{*}\left(W_{j}, W_{j-1} ; \mathbf{Q}\right) \cong H_{*}\left(W_{j} ; \mathbf{Q}\right) / H_{*}\left(W_{j-1} ; \mathbf{Q}\right) .
$$


Proof. We have that the $E^{1}$-term of the spectral sequence above is given as

$$
\begin{aligned}
H_{*}\left(S P^{j}\left(M_{g}\right) ; \mathbf{Q}\right) & \oplus H_{*}\left(S P^{j-2}\left(M_{g}\right) ; \mathbf{Q}\right)\left(1, h_{1}, h_{3}\right) \\
& \oplus \sum_{2 \leq 2 l \leq j} H_{*}\left(S P^{j-2 l}\left(M_{g}\right) ; \mathbf{Q}\right)\left(1, h_{1}, h_{3}, h_{1} h_{3}\right) .
\end{aligned}
$$

$d_{1}\left(\Theta h_{1}\right)=i_{*}(\Theta)-\Theta$ where $\Theta \in H_{*}\left(S P^{j-2 l}\left(M_{g}\right)\right)$ and

$$
i: S P^{j-2 l}\left(M_{g}\right) \rightarrow S P^{j-2 l+2}\left(M_{g}\right)
$$

is a base point inclusion. Similarly

$$
d_{1}\left(\Theta h_{1} h_{3}\right)=d_{1}\left(\Theta h_{1}\right) h_{3} \pm\left(\Theta h_{1}\right) d_{1}\left(h_{3}\right)
$$

where, of course, this last term is just multiplication by $\left[M_{g}\right]-\sum e_{2 i-1} e_{2 i}$. Ignoring the last piece one sees that the $d_{1}$-differential on $h_{1}$ reduces the calculation of $E^{2}$ to the following complex:

$$
\begin{aligned}
H_{*}\left(S P^{j-2}\left(M_{g}\right) ; \mathbf{Q}\right) h_{3} & \longrightarrow H_{*}\left(S P^{j}\left(M_{g}\right) ; \mathbf{Q}\right), \\
\Theta h_{3} & \mapsto \Theta\left(\left[M_{g}\right]-\sum_{1}^{g} e_{2 i-1} e_{2 i}\right)
\end{aligned}
$$

from which the first statement of the lemma follows. The second statement is then immediate. q.e.d.

The remaining groups $H_{*}\left(L E_{v, \ldots, v} ; \mathbf{Q}\right)$ in the hyperelliptic case.

It is convenient to write $v=g+s, 1 \leq s<g-1$ when $v>g$. In this case we have that $J\left(M_{g}\right)$ is filtered by $W_{0} \subset W_{1} \subset \cdots W_{g-1} \subset J\left(M_{g}\right)$, and over points in $W_{g-s-2 l}-W_{g-s-2 l-2}$ the inverse image in $E_{v, \ldots, v}$ is $\left(\mathbf{P}^{s+l}\right)^{n+1}$, while the inverse image over $J\left(M_{g}\right)-W_{g-s-2}$ is $\left(\mathbf{P}^{s}\right)^{n+1}$. Thus, we obtain a spectral sequence with $E^{1}$-term:

$$
\begin{gathered}
H_{*}\left(J\left(M_{g}\right), W_{g-s-2}\right) \otimes\left(H_{*}\left(\mathbf{P}^{s}\right)\right)^{n+1} \\
\oplus \sum_{l=1}^{[g-s / 2]} H_{*}\left(W_{g-s-2 l}, W_{g-s-2 l-2}\right) \otimes\left(H_{*}\left(\mathbf{P}^{s+l}\right)\right)^{n+1} \\
\Longrightarrow H_{*}\left(E_{g+s, \ldots, g+s}\right)
\end{gathered}
$$

with field coefficients. 
Similarly, the corresponding $E^{1}$-term for $H_{*}\left(L E_{g+s, \ldots, g+s}\right)$ is given by

$$
H_{*}\left(J\left(M_{g}\right), W_{g-s-1}\right) \otimes\left(H_{*}\left(\mathbf{P}^{s}, \mathbf{P}^{s-1}\right)\right)^{n+1}
$$

$$
\begin{aligned}
& \oplus \sum_{l=1}^{[g-s / 2]} H_{*}\left(W_{g-s-2 l}, W_{g-s-2 l-1}\right) \otimes\left(H_{*}\left(\mathbf{P}^{s+l}, \mathbf{P}^{s+l-1}\right)\right)^{n+1} \\
& \Longrightarrow H_{*}\left(L E_{g+s, \ldots, g+s}\right) .
\end{aligned}
$$

Note here that in the formula above the term involving the relative groups

$$
H_{*}\left(W_{g-s-2 l-1}, W_{g-s-2 l-2}\right)
$$

is zero. That is because, in this region of $J\left(M_{g}\right)$ and this range for $v$ the inverse image of a point is $\mathbf{P}^{s+l}$ in both $E_{g+s, \ldots, g+s}$ and $E_{g+s-1, \ldots, g+s-1}$.

Again, because of this gap the spectral sequence collapses and $E^{1}=$ $E^{\infty}$. Of course the calculations become considerably more complex with $\mathbf{F}_{p}$ coefficients.

\section{The Ext-groups for $\Lambda_{g} / S_{g}$}

By inspection, in the spectral sequence of (5.9) we find the that $d_{1}$-differential gives rise to a series of complexes that occur in the calculation of $E x t$-groups for the ring $V_{g}$ defined below. In this section we calculate these Ext-groups and apply the results in $\S 15$ to determine the structure of the rational homology of the space $\operatorname{Hol}_{k}^{*}\left(M_{g}, \mathbf{P}^{n}\right)$ with $M_{g}$ hyperelliptic and $n \geq 2$.

Notation 14.1.

(1) $\Lambda_{g}=\Lambda\left(e_{1}, \ldots, e_{2 g}\right)=H_{*}\left(J\left(M_{g}\right), \mathbf{Q}\right)$,

(2) $f_{g}=\sum_{i=1}^{g} e_{2 i-1} e_{2 i} \in \Lambda_{g}$,

(3) $S_{g} \subset \Lambda_{g}$ is the ideal $\left(f_{g}\right)$, and

(4) $\Lambda_{g} / S_{g}=V_{g}$ is the quotient.

$V_{g}$ occurs quite often in both our study of $\operatorname{Hol}_{d}^{*}\left(M_{g}, \mathrm{P}^{n}\right)$ and $\operatorname{Map}_{d}^{*}\left(M_{g}, \mathbf{P}^{n}\right)$. In particular we need the groups $\operatorname{Ext}_{\Lambda_{g}^{* *}}^{*}\left(V_{g}, \mathbf{Q}\right)$ which we will describe as modules over $\operatorname{Ext}_{\Lambda_{g}}^{*, *}(\mathbf{Q}, \mathbf{Q})=\mathbf{Q}\left[h_{1}, \ldots, h_{2 g}\right]$ where $h_{i}=\left|e_{i}\right|^{*}, 1 \leq i \leq 2 g$. 
In [2] it is shown that

$$
* f_{g}:\left(\Lambda_{g}\right)_{i} \longrightarrow\left(\Lambda_{g}\right)_{i+2}
$$

is injective for $i \leq g-1$ and surjective for $i \geq g-1$. Consequently, $\left(V_{g}\right)_{i}=0$ for $i>g$ while $\left(V_{g}\right)_{i}=\mathbf{Q}^{N(g, i)}$ for $0 \leq i<g$ where

$$
N(g, i)= \begin{cases}\left(\begin{array}{c}
2 g \\
i
\end{array}\right)-\left(\begin{array}{c}
2 g \\
i-2
\end{array}\right) & \text { for } 2 \leq i \leq g \\
2 g & \text { for } i=1 \\
1 & \text { for } i=0\end{cases}
$$

As a special case note that

$$
V_{1}=\Lambda_{1} /\left\{e_{1} e_{2}\right\}
$$

so there is a short exact sequence of $\Lambda_{1}$-modules

$$
0 \longrightarrow \mathbf{Q} \stackrel{e_{1} e_{2}}{\leftrightarrow} \Lambda_{1} \longrightarrow V_{1} \longrightarrow 0
$$

where $\mathbf{Q}$ denotes the "trivial" module given by the augmentation map $\epsilon: \Lambda_{g} \rightarrow \mathbf{Q}, \epsilon\left(e_{i}\right)=0, \epsilon(1)=1$. This exact sequence gives rise to the long exact sequence of Ext-groups:

$$
\begin{aligned}
\cdots & \longrightarrow E x t_{\Lambda_{1}}^{i-1, j-2}(\mathbf{Q}, \mathbf{Q}) \stackrel{\delta}{\longrightarrow} E x t_{\Lambda_{1}}^{i, j}\left(V_{1}, \mathbf{Q}\right) \\
& \longrightarrow E x t_{\Lambda_{1}}^{i, j}\left(\Lambda_{1}, \mathbf{Q}\right) \longrightarrow E x t_{\Lambda_{1}}^{i, j}(\mathbf{Q}, \mathbf{Q}) \stackrel{\delta}{\longrightarrow} \cdots,
\end{aligned}
$$

and since $\operatorname{Ext}_{\Lambda_{1}}^{i, j}\left(\Lambda_{1}, \mathbf{Q}\right)=\left\{\begin{array}{ll}0 & (i, j) \neq(0,0), \\ \mathbf{Q} & (i, j)=(0,0),\end{array}\right.$ we have

$$
\begin{aligned}
\operatorname{Ext}_{\Lambda_{1}}^{*, *}\left(V_{1}, \mathbf{Q}\right) & =\mathbf{Q} e_{0,0} \oplus E x t_{\Lambda_{1}}^{*, *}(\mathbf{Q}, \mathbf{Q}) P_{1,2} \\
& =\mathbf{Q} e_{0,0} \oplus \mathbf{Q}\left[h_{1}, h_{2}\right] P_{1,2},
\end{aligned}
$$

where $P_{1,2}$ is a generating element in $\operatorname{Ext}_{\Lambda_{1}}^{1,2}\left(V_{1}, \mathbf{Q}\right)$.

Before we can describe the structure of the modules $\operatorname{Ext}_{\Lambda_{g}}^{*, *}\left(V_{g}, \mathbf{Q}\right)$ for $g>1$, we need some preliminary constructions.

First, we will often be dealing with a situation where we have a ring $R_{i+1}=R_{i} \otimes_{\mathrm{Q}} R_{1}$ given as the tensor product of augmented rings, together with a module, $M$, over $R_{i}$. $M$ becomes a module over $R_{i+1}$ via the following obvious composition

$\left(R_{i} \otimes R_{1}\right) \otimes M \stackrel{i d \otimes i d}{\longrightarrow}\left(R_{i} \otimes R_{1}\right) \otimes(M \otimes \mathbf{Q} \mathbf{Q}) \stackrel{1 \otimes T \otimes 1}{\longrightarrow}\left(R_{i} \otimes M\right) \otimes\left(R_{1} \otimes \mathbf{Q}\right)$ $\stackrel{\mu_{i} \otimes \epsilon}{\longrightarrow} M \otimes \mathbf{Q} \mathbf{Q}=M$. 
Then, by change of rings,

$$
\operatorname{Ext}_{R_{i+1}}(M, \mathbf{Q})=\operatorname{Ext}_{R_{i}}(M, \mathbf{Q}) \otimes \operatorname{Ext}_{R_{1}}(\mathbf{Q}, \mathbf{Q})
$$

Second, we will need to consider modules of the following kind: given a module $M$ over the ring $R$ and an exact sequence of $R$-modules

$$
0 \longrightarrow K \hookrightarrow(R)^{N} \longrightarrow M \longrightarrow 0,
$$

then $K$ is written $\Omega M$ and is unique up to direct sum with a free $R$ module. The following result is well known.

Lemma 14.3. $\operatorname{Ext}_{R}^{i, j}(\Omega M, N) \cong \operatorname{Ext}_{R}^{i+1, j}(M, N)$ for all $i \geq 1$, and

$$
\operatorname{Ext}_{R}^{0, *}(\Omega M, N) \rightarrow \operatorname{Ext}_{R}^{1, *}(M, N)
$$

is surjective for all $R$-modules $N$.

We say $\Omega M$ is minimal if the natural map

$$
\operatorname{Ext}_{R}^{0, *}(\Omega M, \mathbf{Q}) \rightarrow \operatorname{Ext}_{R}^{1, *}(M, \mathbf{Q})
$$

is an isomorphism as well. Of course, $\Omega M$ is minimal if and only if the map $R^{N} \rightarrow M$ induces isomorphisms of $E x t^{0, *}(, \mathrm{Q})$-groups.

Consider the following module:

$$
M_{1}=I \subset \mathbf{Q}\left[h_{1}, h_{2}\right]
$$

it is the augmentation ideal, $I=\left(h_{1}, h_{2}\right)$. Moreover, $M_{1}=\Omega \mathbf{Q}$ and is even minimal for $R=\mathbf{Q}\left[h_{1}, h_{2}\right]$; thus

$$
\operatorname{Ext}_{\mathbf{Q}\left[h_{1}, h_{2}\right]}^{i, j}\left(M_{1}, \mathbf{Q}\right)=\operatorname{Ext}_{\mathbf{Q}\left[h_{1}, h_{2}\right]}^{i+1, j}(\mathbf{Q}, \mathbf{Q})
$$

for all $j$ and $i \geq 0$. Moreover, $\operatorname{Ext}_{\mathbf{Q}\left[h_{1}, h_{2}\right]}(\mathbf{Q}, \mathbf{Q})=\Lambda\left(\left|h_{1}\right|^{*},\left|h_{2}\right|^{*}\right)$; thus

$$
\operatorname{Ext}_{\mathbf{Q}\left[h_{1}, h_{2}\right]}^{i, j}\left(M_{1}, \mathbf{Q}\right)= \begin{cases}\mathbf{Q}\left\{\left|h_{1}\right|^{*},\left|h_{2}\right|^{*}\right\} & \text { if }(i, j)=(0,2), \\ \mathbf{Q}\left\{\left|h_{1}\right|^{*}\left|h_{2}\right|^{*}\right\} & \text { if }(i, j)=(1,4) .\end{cases}
$$

It follows that a minimal resolution for $M_{1}$ over $\mathbf{Q}\left[h_{1}, h_{2}\right]$ has the form

$$
\mathbf{Q}\left[h_{1}, h_{2}\right] h_{1,2} \longrightarrow \mathbf{Q}\left[h_{1}, h_{2}\right]\left\{\left[h_{1}\right],\left[h_{2}\right]\right\} \longrightarrow M_{1} \longrightarrow 0,
$$

where $\left[h_{i}\right] \mapsto h_{i}$ and $h_{1,2} \mapsto h_{1}\left[h_{2}\right]-h_{2}\left[h_{1}\right]$. 
Next we consider $M_{1}$ as a module over

$$
\mathbf{Q}\left[h_{1}, h_{2}, h_{3}, h_{4}\right]=\mathbf{Q}\left[h_{1}, h_{2}\right] \otimes \mathbf{Q}\left[h_{3}, h_{4}\right]
$$

as above, and define $M_{2}$ as the kernel of the surjective homomorphism

$$
\pi_{2}: \mathbf{Q}\left[h_{1}, h_{2}, h_{3}, h_{4}\right]\left\{b_{1}, b_{2}\right\} \longrightarrow M_{1} \longrightarrow 0,
$$

$\pi_{2}\left(b_{i}\right)=h_{i}$. Clearly $M_{2}=\Omega M_{1}$ as a module over $\mathbf{Q}\left[h_{1}, h_{2}, h_{3}, h_{4}\right]$ and is minimal as well. Consequently we have

$$
\begin{aligned}
& \operatorname{Ext}_{\mathbf{Q}\left[h_{1}, \ldots, h_{4}\right]}^{0, *}\left(M_{2}, \mathbf{Q}\right)=\operatorname{Ext}_{\mathbf{Q}\left[h_{1}, \ldots, h_{4}\right]}^{1, *}\left(M_{1}, \mathbf{Q}\right) \\
& =\operatorname{Ext}_{\mathbf{Q}\left[h_{1}, h_{2}\right]}^{2, *}(\mathbf{Q}, \mathbf{Q}) \oplus \operatorname{Ext}_{\mathbf{Q}\left[h_{1}, h_{2}\right]}^{1, *}(\mathbf{Q}, \mathbf{Q}) \\
& \otimes \operatorname{Ext}_{\mathbf{Q}\left[h_{3}, h_{4}\right]}^{1, *}(\mathbf{Q}, \mathbf{Q}) \\
& =\mathbf{Q}^{5}
\end{aligned}
$$

with generators $\left|h_{1}\right|^{*}\left|h_{2}\right|^{*},\left|h_{1}\right|^{*}\left|h_{3}\right|^{*},\left|h_{1}\right|^{*}\left|h_{4}\right|^{*},\left|h_{2}\right|^{*}\left|h_{3}\right|^{*},\left|h_{2}\right|^{*}\left|h_{4}\right|^{*}$. Likewise,

$$
\begin{aligned}
\operatorname{Ext}^{1}\left(M_{2}, \mathbf{Q}\right) & =\operatorname{Ext}^{2}\left(M_{1}, \mathbf{Q}\right) \\
& \cong \operatorname{Ext}_{\mathbf{Q}\left[h_{1}, h_{2}\right]}^{2}(\mathbf{Q}, \mathbf{Q}) \otimes \operatorname{Ext}_{\mathbf{Q}\left[h_{3}, h_{4}\right]}^{1}(\mathbf{Q}, \mathbf{Q}) \\
& \quad \oplus \operatorname{Ext}_{\mathbf{Q}\left[h_{1}, h_{2}\right]}^{1}(\mathbf{Q}, \mathbf{Q}) \otimes \operatorname{Ext}_{\mathbf{Q}\left[h_{3}, h_{4}\right]}^{1}(\mathbf{Q}, \mathbf{Q}) \\
& =\mathbf{Q}^{4}
\end{aligned}
$$

To obtain $M_{3}$ regard $M_{2}$ as a module over $\mathbf{Q}\left[h_{1}, \ldots, h_{6}\right]=R_{3}$, and define $M_{3}=\Omega M_{2}$, the kernel in the exact sequence

$$
0 \longrightarrow M_{3} \hookrightarrow R_{3}^{5} \longrightarrow M_{2} \longrightarrow 0,
$$

where the map to $M_{2}$ takes the basis for $R_{3}^{5}$ to the five generators of $M_{2}$ corresponding to the description above. $M_{3}$ is again minimal.

In general $M_{n}$ is given as a module over $R_{n}=\mathbf{Q}\left[h_{1}, \ldots, h_{2 n}\right]$, indeed is a submodule of a free $R_{n}$-module, and $M_{n+1}$ is $\Omega M_{n}$ where $M_{n}$ is now regarded as a module over $R_{n+1}=R_{n} \otimes \mathbf{Q}\left[h_{2 n+1}, h_{2 n+2}\right]$.

Note that for $M_{1}$ we have $\operatorname{Ext}^{0}\left(M_{1}, \mathbf{Q}\right)=\operatorname{Ext}^{0,2}\left(M_{1}, \mathbf{Q}\right)$, and for $M_{2}, E x t^{0}\left(M_{2}, \mathbf{Q}\right)=E x t^{0,4}\left(M_{2}, \mathbf{Q}\right)$. In other words, for these modules $E x t^{0}$ is concentrated in a single bidegree. Also, note that $\operatorname{Ext}_{R_{n}}(\mathbf{Q}, \mathbf{Q})=$ $\Lambda\left(\left|h_{1}\right|^{*}, \ldots,\left|h_{2 n}\right|^{*}\right)$ has each generator $\left|h_{i}\right|^{*}$ occurring in bidegree $(1,2)$, 
so all the elements in $E x t^{i}$ occur in a single bidegree $(i, 2 i)$ here as well. In fact we have by a direct induction

Lemma 14.5. The action map

$$
\operatorname{Ext}_{R_{n}}^{*, *}(\mathbf{Q}, \mathbf{Q}) \otimes \operatorname{Ext}_{R_{n}}^{0, *}\left(M_{n}, \mathbf{Q}\right) \longrightarrow E_{x} t_{R_{n}}^{i, j}\left(M_{n}, \mathbf{Q}\right)
$$

is surjective in all degrees, and $\operatorname{Ext}_{R_{n}}^{0, j}\left(M_{n}, \mathbf{Q}\right)=0$ unless $j=2 n$. Consequently,

$$
\operatorname{Ext}_{R_{n}}^{i, j}\left(M_{n}, \mathbf{Q}\right)=0
$$

unless $j=2 n+2 i$.

As an immediate corollary we see that the $M_{i}$, each being minimal, are all unique, since, in as much as all the generators of $M_{i}$ occur in the same degree, the map of the minimal free module onto $M_{i}$ is well defined up to an isomorphism, hence the same is true of the kernel, $M_{i+1}$.

With this backround discussion complete we are able to state our main result.

Theorem 14.6. Suppose $g \geq 2$. Then

$$
\operatorname{Ext}_{\Lambda_{\boldsymbol{g}}}^{i, j}\left(V_{\boldsymbol{g}}, \mathbf{Q}\right)= \begin{cases}\mathbf{Q} & \text { if }(i, j)=(0,0), \\ \mathbf{Q} & \text { if }(i, j)=(1,2), \\ \left(M_{\boldsymbol{g}}\right)_{i-2, j+i-4} & \text { if } i \geq 2 .\end{cases}
$$

In particular, as a module over $\operatorname{Ext}_{\Lambda_{g}}(\mathbf{Q}, \mathbf{Q})$

$$
\operatorname{Ext}_{\Lambda_{g}}\left(V_{g}, \mathbf{Q}\right)=\mathbf{Q} \oplus \mathbf{Q} \oplus M_{g}
$$

where the first generator gives $E x t^{0,0}$, the second gives $E x t^{1,2}$ and the rest is shifted by $(2,-i+4)$.

Example 14.7. We have

$$
\operatorname{Ext}_{\Lambda_{2}}\left(V_{2}, \mathbf{Q}\right)=\mathbf{Q} e_{0,0} \oplus \mathbf{Q} P_{1,2} \oplus \mathbf{Q}\left[h_{1}, \ldots, h_{4}\right]\left\{b_{1}, b_{2}, \ldots, b_{5}\right\} / \mathcal{R},
$$

where $b_{i} \in \operatorname{Ext}_{\Lambda_{2}}^{2,4}\left(V_{2}, \mathbf{Q}\right)$, and $\mathcal{R}$ is the set of four relations

$$
\mathcal{R}=\left\{h_{5} b_{1}, h_{6} b_{1}, h_{5} b_{3}-h_{6} b_{2}, h_{5} b_{5}-h_{6} b_{4}\right\} .
$$

Proof. A resolution of $V_{g}$ over $\Lambda_{g}$ starts in the following way

$$
0 \longrightarrow K_{g} \hookrightarrow \Lambda_{g} P_{1,2} \stackrel{* f_{g}}{\longrightarrow} \Lambda_{g} e_{0,0} \longrightarrow V_{g} \longrightarrow 0,
$$


and we have already seen that $\left(K_{g}\right)_{i}=0$ for $i \leq g+1$. Thus it follows that

$$
\operatorname{Ext}_{\Lambda_{g}}^{r, s}\left(V_{g}, \mathbf{Q}\right)=0 \text { whenever } r \geq 2 \text { and } s \leq g+1 .
$$

On the other hand, since $K_{g}$ has $N(g, g)$ generators in $g+2$, we have that

$$
\operatorname{Ext}_{\Lambda_{g}}^{2, g+2}\left(V_{g}, \mathbf{Q}\right)=\mathbf{Q}^{N(g, g)} .
$$

We now give an inductive analysis of the $V_{g}$. In particular we assume the theorem is true for $V_{g-1}$ and begin by establishing a relation between $V_{g}$ and $V_{g-1}$. Actually, we start with $g=1$ where the theorem is not quite true as stated, but $\operatorname{Ext}_{\Lambda_{1}}\left(V_{1}, \mathbf{Q}\right)$ is given as an extension

$$
0 \longrightarrow M_{1} \longrightarrow \operatorname{Ext}_{\Lambda_{1}}\left(V_{1}, \mathbf{Q}\right) \longrightarrow \mathbf{Q} e_{0,0} \oplus \mathbf{Q} P_{1,2} \longrightarrow 0,
$$

which turns out to be sufficient to start the induction. There is a surjection

$$
\pi_{g}: V_{g} \longrightarrow V_{g-1}
$$

induced by the projection $p_{g}: \Lambda_{g} \rightarrow \Lambda_{g-1}$,

$$
p_{g}\left(e_{i}\right)= \begin{cases}e_{i} & \text { for } i \leq i \leq 2 g-2, \\ 0 & \text { for } \mathrm{i}=2 \mathrm{~g}-1 \text { or } i=2 g,\end{cases}
$$

which takes $f_{g}$ to $f_{g-1}$. Let $N_{g}$ be the kernel of $\pi_{g}$, and $\mathcal{K}_{g}$ be the kernel of $p_{g}$. Then we can write

$$
\mathcal{K}_{g} \cong \Lambda_{g-1}\left\{e_{2 g-1}, e_{2 g}, e_{2 g-1} e_{2 g}\right\}
$$

and

$$
\mathcal{K}_{g} / f_{g} \mathcal{K}_{g}=V_{g-1} \otimes \mathbf{Q}\left\{e_{2 g-1}, e_{2 g}, e_{2 g-1} e_{2 g}\right\}
$$

as a module over $\Lambda_{g}$. However, the surjection

$$
\bar{p}_{g}: \mathcal{K}_{g} / f_{g} \mathcal{K}_{g} \longrightarrow N_{g} \longrightarrow 0
$$

is not an isomorphism. In fact we have

Lemma 14.8. The kernel of $\bar{p}_{g}$ is a direct sum of trivial modules over $\Lambda_{g}$ concentrated in degree $g+1$,

$$
\operatorname{Ker}\left(\bar{p}_{g}\right)=\operatorname{Ker}\left(\bar{p}_{g}\right)_{g+1} \cong \mathbf{Q}^{N(g-1, g-1)}
$$

as a module over $\Lambda_{g}$. 
Proof. For $2 \leq i \leq g-1$, using the expansion

$$
\left(\begin{array}{l}
r \\
i
\end{array}\right)=\left(\begin{array}{c}
r-2 \\
i
\end{array}\right)+2\left(\begin{array}{c}
r-2 \\
i-1
\end{array}\right)+\left(\begin{array}{c}
r-2 \\
i-2
\end{array}\right)
$$

valid for $r \geq i \geq 2$, we have

$$
\begin{aligned}
\operatorname{Dim}\left(N_{g}\right)_{i} & =\left[\left(\begin{array}{c}
2 g \\
i
\end{array}\right)-\left(\begin{array}{c}
2 g \\
i-2
\end{array}\right)\right]-\left[\left(\begin{array}{c}
2 g-2 \\
i
\end{array}\right)-\left(\begin{array}{c}
2 g-2 \\
i-2
\end{array}\right)\right] \\
& =\left\{2\left(\begin{array}{c}
2 g-2 \\
i-1
\end{array}\right)+\left(\begin{array}{c}
2 g-2 \\
i-2
\end{array}\right)\right\}-\left\{2\left(\begin{array}{c}
2 g-2 \\
i-3
\end{array}\right)+\left(\begin{array}{c}
2 g-2 \\
i-4
\end{array}\right)\right\} .
\end{aligned}
$$

This shows that $\bar{p}_{g}$ is an isomorphism in this range. Likewise,

$$
\begin{aligned}
\operatorname{Dim}\left(N_{g}\right)_{g} & =\left(\begin{array}{c}
2 g \\
g
\end{array}\right)-\left(\begin{array}{c}
2 g \\
g-2
\end{array}\right) \\
& =\left(\begin{array}{c}
2 g-2 \\
g
\end{array}\right)+2\left(\begin{array}{c}
2 g-2 \\
g-1
\end{array}\right)-2\left(\begin{array}{c}
2 g-2 \\
g-3
\end{array}\right)-\left(\begin{array}{c}
2 g-2 \\
g-4
\end{array}\right) \\
& =\left[\left(\begin{array}{c}
2 g-2 \\
g-2
\end{array}\right)-\left(\begin{array}{c}
2 g-2 \\
g-4
\end{array}\right)\right]-2\left[\left(\begin{array}{c}
2 g-2 \\
g-1
\end{array}\right)-\left(\begin{array}{c}
2 g-2 \\
g-3
\end{array}\right)\right]
\end{aligned}
$$

using the equality $\left(\begin{array}{c}2 g-2 \\ g\end{array}\right)=\left(\begin{array}{c}2 g-2 \\ g-2\end{array}\right)$, which gives the isomorphism in dimension $g$. However, $\left(N_{g}\right)_{g+1}=0$ while

$$
\operatorname{Dim}\left(\left(\mathcal{K}_{g} / f_{g} \mathcal{K}_{g}\right)_{g+1}\right)=\operatorname{Dim}\left(\left(V_{g-1}\right)_{g-1}\right)=N(g-1, g-1),
$$

and the lemma follows since $\left(\mathcal{K}_{g} / f_{g} \mathcal{K}_{g}\right)_{t}=0$ for $t \geq g+2$. q.e.d.

Consequently we have a short exact sequence of $\Lambda_{g}$-modules

$$
0 \longrightarrow \mathrm{Q}^{N(g-1, g-1)} \longrightarrow \mathcal{K}_{g} / f_{g} \mathcal{K}_{g} \longrightarrow N_{g} \longrightarrow 0
$$

and a long exact sequence of Ext-modules

$$
\begin{gathered}
\cdots \stackrel{\delta}{\longrightarrow} \operatorname{Ext}_{\Lambda_{g}}\left(N_{g}, \mathbf{Q}\right) \longrightarrow E x t_{\Lambda_{g}}\left(\mathcal{K}_{g}, \mathbf{Q}\right) \\
\stackrel{i^{*}}{\longrightarrow} \coprod_{i=1}^{N(g-1, g-1)} \operatorname{Ext}_{\Lambda_{g}}(\mathbf{Q}, \mathbf{Q}) \chi_{i} \stackrel{\delta}{\longrightarrow} \cdots
\end{gathered}
$$

where the generator, $\chi_{i}$ for each of the $\operatorname{Ext}_{\Lambda_{g}}(\mathbf{Q}, \mathbf{Q})$ terms occurs in bidegree $(0, g+1)$. 
By change of rings we have

$$
\begin{aligned}
\operatorname{Ext}_{\Lambda_{g}}\left(\mathcal{K}_{g}, \mathbf{Q}\right) \cong & \operatorname{Ext}_{\Lambda_{g-1}}\left(V_{g-1}, \mathbf{Q}\right) \\
& \quad \oplus \operatorname{Ext}_{\Lambda\left(e_{2 g-1}, e_{2 g}\right)}\left(\left\{e_{2 g-1}, e_{2 g}, e_{2 g-1} e_{2 g}\right\}, \mathbf{Q}\right) \\
= & \operatorname{Ext}_{\Lambda_{g-1}}\left(V_{g-1}, \mathbf{Q}\right) \otimes \mathbf{Q}\left[h_{2 g-1}, h_{2 g}\right]\left(b_{1}, b_{2}\right) / \mathcal{R}
\end{aligned}
$$

where $\mathcal{R}$ is the relation $h_{2 g} b_{1}-h_{2 g-1} b_{2}=0$. By our inductive assumption this implies that the generators of $\operatorname{Ext}_{\Lambda_{g}}\left(\mathcal{K}_{g}, \mathbf{Q}\right)$ as a module over $\operatorname{Ext}_{\Lambda_{g}}(\mathbf{Q}, \mathbf{Q})$ occur in bidegrees $(0,1),(1,3)$, and $(2, g+2)$. But in these bidegrees $\operatorname{Ext}_{\Lambda_{g}}(\mathbf{Q}, \mathbf{Q}) e_{0, g+1}$ is identically zero. It follows that the map $i^{*}$ in $(*)$ is zero so

$$
\delta: \quad \coprod_{i=1}^{N(g-1, g-1)} E x t_{\Lambda_{g}}(\mathbf{Q}, \mathbf{Q}) \chi_{i} \longrightarrow \operatorname{Ext}_{\Lambda_{g}}\left(N_{g}, \mathbf{Q}\right)
$$

is an injection, and therefore

$$
\operatorname{Ext}_{\Lambda_{g}}\left(N_{g}, \mathbf{Q}\right)=\coprod_{i=1}^{N(g-1, g-1)} \operatorname{Ext}_{\Lambda_{g}}(\mathbf{Q}, \mathbf{Q}) e_{1, g+1}^{i} \oplus E x t_{\Lambda_{g}}\left(\mathcal{K}_{g}, \mathbf{Q}\right)
$$

as modules over $\operatorname{Ext}_{\Lambda_{g}}(\mathbf{Q}, \mathbf{Q})$ up to a possible extension problem.

The remainder of the proof is direct. The exact sequence

$$
0 \longrightarrow N_{g} \longrightarrow V_{g} \stackrel{\pi_{g}}{\longrightarrow} V_{g-1} \longrightarrow 0
$$

gives us the long exact sequence of Ext-groups

$$
\begin{aligned}
\cdots E x t_{\Lambda_{g}}^{i}\left(N_{g}, \mathbf{Q}\right) \stackrel{\delta}{\longrightarrow} \operatorname{Ext}_{\Lambda_{g-1}}^{i+1}\left(V_{g-1}, \mathbf{Q}\right) \otimes \mathbf{Q}\left[h_{2 g-1}, h_{2 g}\right] \\
\\
\longrightarrow \operatorname{Ext}_{\Lambda_{g}}\left(V_{g}, \mathbf{Q}\right) \longrightarrow \cdots
\end{aligned}
$$

The map $\delta$ on the piece $\operatorname{Ext}_{\Lambda_{g}}\left(\mathcal{K}_{g}, \mathbf{Q}\right)$ in $\operatorname{Ext}_{\Lambda_{g}}\left(N_{g}, \mathbf{Q}\right)$ injects to

$$
\operatorname{Ext}_{\Lambda_{g-1}}\left(V_{g-1}, \mathbf{Q}\right) \otimes I
$$

where $I \subset \mathbf{Q}\left[h_{2 g-1}, h_{2 g}\right]$ is the augmentation ideal $\left(h_{2 h-1}, h_{2 g}\right)$. The resulting quotient is $\operatorname{Ext}_{\Lambda_{g-1}}\left(V_{g-1}, \mathbf{Q}\right)$ and so the calculation reduces to the determination of the map

$$
\delta: \quad \coprod_{i=1}^{N(g-1, g-1)} E x t_{\Lambda_{g}}(\mathbf{Q}, \mathbf{Q}) \chi_{i} \longrightarrow M_{g-1}
$$


from the inductive assumption. However, we know $\operatorname{Ext}_{\Lambda_{g}}^{*, j}\left(V_{g}, \mathbf{Q}\right)=0$ for $* \geq 2$ and $j \leq g+1$, and also

$$
\operatorname{Ext}_{\Lambda_{g-1}}^{2, g+1}\left(V_{g-1}, \mathbf{Q}\right)=\mathbf{Q}^{N(g-1, g-1)},
$$

so it follows that $\delta$ must give an isomorphism from $(1, g+1)$ to $(2, g+1)$. Since $M_{g-1}$ is generated over $\operatorname{Ext}_{\Lambda_{g-1}}(\mathbf{Q}, \mathbf{Q})$ by the elements in this dimension, consequently $\delta$ is surjective and the kernel is minimal and hence $M_{g}$, with the generators all occuring in bidegree $(2, g+2)$. The induction is complete. q.e.d.

\section{The rational homology of $\operatorname{Hol}_{k}^{*}\left(M_{g}, \mathbf{P}^{n}\right)$ for $M_{g}$ hyperelliptic}

We now use the results of $\S 10-\S 14$ to prove the following theorem.

Theorem 15.1. The map

$$
H_{*}\left(\operatorname{Hol}_{k}^{*}\left(M_{g}, \mathbf{P}^{n}\right) ; \mathbf{Q}\right) \rightarrow H_{*}\left(\operatorname{Map}_{k}^{*}\left(M_{g}, \mathbf{P}^{n}\right) ; \mathbf{Q}\right)
$$

is injective for $k \geq 2 g$ and $n>2$.

Proof. Consider the subquotient of the $E^{1}$ term in the spectral sequence of (5.9) converging to $H_{*}\left(Q E_{v, \ldots, v} ; \mathbf{Q}\right)$ defined as the direct sum

$$
\begin{aligned}
& \sum_{v=2 s}^{g+s-1} H_{*}\left(W_{v-2 s}, W_{v-2 s-1}\right) \otimes \tilde{H}_{*}\left(S^{2 s(n+1)}\right) \\
& \otimes H_{*}\left(S P^{k-v}\left(\Sigma M_{g}\right), S P^{k-v-1}\left(\Sigma M_{g}\right) ; \mathbf{Q}\right)
\end{aligned}
$$

for a given $s=0,1,2, \ldots$ with the term

$$
\begin{aligned}
& H_{*}\left(J\left(M_{g}\right), W_{g-s+1}\right) \otimes \tilde{H}_{*}\left(S^{2 s(n+1)}\right) \\
& \quad \otimes H_{*}\left(S P^{k-g-s}\left(\Sigma M_{g}\right), S P^{k-v-s-1}\left(\Sigma M_{g}\right) ; \mathbf{Q}\right)
\end{aligned}
$$

added but playing a special role. We have determined that

$$
H_{*}\left(W_{j}, W_{j-1}\right)=\sum_{l=0}^{[j / 2]}\left(V_{g}\right)_{j-2 l}[M]^{l},
$$

where $[M] \sim \sum_{1}^{g} e_{2 i-1} e_{2 i}$ in $H_{*}\left(J\left(M_{g}\right)\right)$. Also,

$$
\begin{aligned}
& H_{*}\left(S P^{k}\left(\Sigma M_{g}\right), S P^{k-1}\left(\Sigma M_{g}\right)\right) \\
& \quad=\mathbf{Q}\left[\left|e_{1}\right|, \ldots,\left|e_{2 g}\right|\right]_{k} \oplus \mathbf{Q}\left[\left|e_{1}\right|, \ldots,\left|e_{2 g}\right|\right]_{k-1}\left|\left[M_{g}\right]\right|
\end{aligned}
$$


and the (sub-quotient) $d_{1}$ differentials are given by $d_{1}\left(\left|\left[M_{g}\right]\right|\right)=\left[M_{g}\right]$, $d_{1}\left(\left|e_{i}\right|\right)=e_{i}$, including the map from the term $H_{*}\left(W_{g-s+1}, W_{g-s}\right) \otimes \cdots$ to the extreme term

$$
H_{*}\left(J\left(M_{g}\right), W_{g-s}\right) \otimes \cdots .
$$

If we first apply the differential to $\left|\left[M_{g}\right]\right|$, we obtain the complex

$$
\left\{\sum_{v=2 s}^{g+s-1}\left(V_{g}\right)_{v-2 s} \otimes \mathbf{Q}\left[\left|e_{1}\right|, \ldots,\left|e_{2 g}\right|\right]_{k-v}\right\} \otimes \tilde{H}_{*}\left(S^{2 s(n+1)}\right),
$$

with differentials as specified as well as an appropriate quotient of the extreme term with differentials mapping to it from the $v=g+s-1$-term in the sum above.

Note that the complex in brackets above is a piece of a direct summand,

$$
\sum_{w=0}^{g-2}\left(V_{g}\right)_{w} \otimes \mathbf{Q}\left[\left|e_{1}\right|, \ldots,\left|e_{2 g}\right|\right]_{k-2 s-w}
$$

of the complex $\left(V_{g}\right) \otimes \mathbf{Q}\left[\left|e_{1}\right|, \ldots,\left|e_{2 g}\right|\right]$ which has, as its homology the groups

$$
\operatorname{Tor}_{*}^{\Lambda_{g}}\left(\mathbf{Q}, V_{g}\right)
$$

Here, the extreme term contains the next term in the resolution as a direct summand, but contains $H_{*}\left(J\left(M_{g}\right), W_{g-s-1}\right) \otimes \cdots$ as well as the complementary summand. On the other hand we have already determined these Tor-groups in $\S 14$ and have shown, in particular that $\operatorname{Tor}_{l, m}^{\Lambda_{g}}\left(\mathbf{Q}, V_{g}\right)=0$ unless $m=m(g, l)$ is

$$
m(g, l)= \begin{cases}l+g & \text { if } l \geq 2 \\ 2 & \text { if } l=1 \\ 0 & \text { if } l=0\end{cases}
$$

But, in the main part of the complex the m's which appear are always less than $m(g, l)$ so they contribute nothing by $E^{2}$, while at $E^{2}$ the term $H_{*}\left(J\left(M_{g}\right), W_{g-s}\right) \otimes \cdots$, which is a surjective image of the stable term $\tilde{H}\left(J\left(M_{g}\right)\right) \otimes \cdots$, contributes a quotient, consequently still a surjective image of the stable term, and the result follows for $n>2$ by the collapsing of the stable spectral sequence at $E^{2}$. q.e.d. 


\section{References}

[1] E. Arbarello, M. Cornalba, P.A. Griffiths \& J. Harris, Geometry of algebraic curves Volume I, Grundlehren, Math. Wiss. Vol 267, Springer, New York, 1985.

[2] C.-F. Bödigheimer, F. R. Cohen \& R. J. Milgram, Truncated symmetric products and configuration spaces, Math. Z. 214 (1993) 179-216.

[3] C. Boyer, J. Hurtubise, B. M. Mann \& R. J. Milgram, The topology of the space of rational maps into generalized flag manifolds, Acta Math. 173 (1994) 61-101.

[4] H. Cartan, Seminaire Henri Cartan, 1954-55, exposes 2-11.

[5] F. R. Cohen, R.L. Cohen, B. M. Mann \& R. J. Milgram, The topology of rational functions and divisors of surfaces, Acta. Math. 166 (1991) 163-221.

[6] A. Dold, Homology of symmetric products and other functors of complexes, Ann. of Math. 68 (1958) 54-80.

[7] A. Dold \& R. Thom, Quasifaserungen und unendliche symmetrische Produkte, Ann. of Math. 67 (1958) 239-281.

[8] P. Griffiths \& J. Harris, Principles of algebraic geometry, Wiley-Interscience, New York, 1978.

[9] M. A. Guest, The topology of the space of rational curves on a toric variety, Acta Math. 174 (1995) 119-145.

[10] R. C. Gunning, Lectures on Riemann surfaces: Jacobi varieties, Princeton Univ. Press, Princeton, 1972.

[11] J. Hurtubise, Holomorphic maps of a Riemann surface into a flag manifold, J. Differential Geom. 43 (1996) 99-118.

[12] S. Kallel, Divisor spaces on punctured Riemann surfaces, to appear in Trans. Amer. Math. Soc.

[13] - The topology of maps from a Riemann surface into complex projective space, Preprint.

[14] T. Kudo, A transgression theorem, Mem. Fac. Sci., Kyushu Univ. Ser. A, 9 (1956) $79-81$.

[15] I. G. Macdonald, Symmetric products of an algebraic curve, Topology 1 (1962) 319-343.

[16] A. Mattuck, Picard bundles, Illinois J. Math. 5 (1961) 550-564.

[17] B. M. Mann \& R. J. Milgram, Some spaces of holomorphic maps to flag manifolds, J. Differential Geom. 33 (1991) 301-324. 
[18] On the moduli space of $S U(n)$ monopoles and holomorphic maps to flag manifolds, J. Differential Geom. 38 (1993) 39-103.

[19] R. J. Milgram, The homology of symmetric products, Trans. Amer. Math. Soc. 138 (1969) 251-265.

[20] _ The geometry of Toeplitz matrices, Topology 36 (1997) 1155-1192.

[21] G. Segal, The topology of spaces of rational functions, Acta. Math. 143 (1979) 39-72.

UNIVERSITÉ DE MONTRÉAL STANFORD UNIVERSITY 